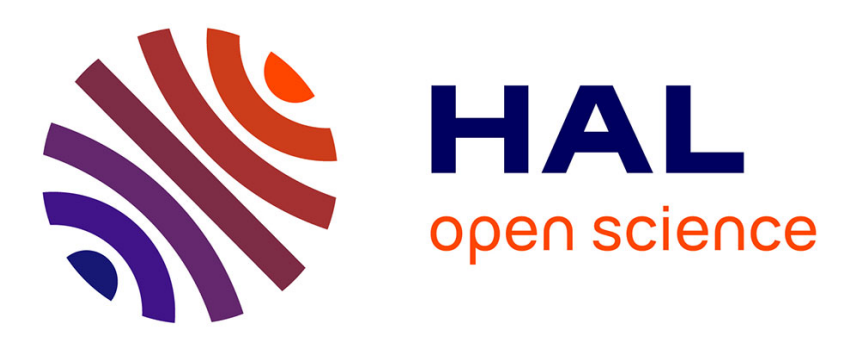

\title{
Microscopic description of rotational spectra including band-mixing. - I. Formulation in a microscopic basis
}

\author{
F. Brut, S. Jang
}

\section{To cite this version:}

F. Brut, S. Jang. Microscopic description of rotational spectra including band-mixing.

I. Formulation in a microscopic basis. Journal de Physique, 1982, 43 (11), pp.1575-1595. 10.1051/jphys:0198200430110157500 . jpa-00209537

\section{HAL Id: jpa-00209537 https://hal.science/jpa-00209537}

Submitted on 1 Jan 1982

HAL is a multi-disciplinary open access archive for the deposit and dissemination of scientific research documents, whether they are published or not. The documents may come from teaching and research institutions in France or abroad, or from public or private research centers.
L'archive ouverte pluridisciplinaire HAL, est destinée au dépôt et à la diffusion de documents scientifiques de niveau recherche, publiés ou non, émanant des établissements d'enseignement et de recherche français ou étrangers, des laboratoires publics ou privés. 
Classification

Physics Abstracts

$21.10-21.60$

\title{
Microscopic description of rotational spectra including band-mixing. I. Formulation in a microscopic basis
}

\author{
F. Brut and S. Jang \\ Institut des Sciences Nucléaires, 53, avenue des Martyrs, 38026 Grenoble Cedex, France
}

(Reçu le 24 mai 1982, accepté le 5 juillet 1982)

\begin{abstract}
Résumé. - La théorie de projection du mouvement collectif est utilisée pour décrire l'énergie de rotation avec mélanges de bandes. L'énergie est écrite comme un développement perturbatif en fonction des puissances inverses d'une quantité reliée à la valeur moyenne de l'opérateur $J_{y}^{2}$. Le présent approche est discuté en relation avec les différences sur les fonctions d'onde individuelles obtenues par résolution des équations variationnelles qui sont écrites avant ou après projection. En plus des différentes quantités familières qui apparaissent dans la formule phénoménologique de l'énergie, tels que le paramètre d'inertie, le facteur de découplage et l'élément de matrice de mélange pour $|\Delta K|=1$, d'autres quantités moins familières ayant les facteurs de phase particulièrs, $(-1)^{J+1} J(J+1),(-1)^{J+3 / 2}\left(J-\frac{1}{2}\right)\left(J+\frac{1}{2}\right)\left(J+\frac{3}{2}\right),(-1)^{J+1 / 2}\left(J+\frac{1}{2}\right) J(J+1),(-1)^{J} J(J+1)(J-1)(J+2)$ et $[J(J+1)]^{2}$ sont également obtenues. Le terme de mélange de bandes avec $|\Delta K|=2$ est aussi nouveau. Toutes ces quantités sont exprimées en fonction de l'interaction à deux corps et des valeurs moyennes de l'opérateur $J_{y}^{m}$, où $m$ est entier, en utilisant le formalisme particule-trou. La différence entre les moments d'inertie d'un noyau pairpair et du noyau pair-impair voisin, aussi bien que les effets du mélange de bandes sur le moment d'inertie sont étudiés. Tous ces résultats sont présentés dans le but de faciliter les comparaisons avec les termes phénoménologiques correspondants et de permettre leur utilisation dans des applications futures.
\end{abstract}

\begin{abstract}
Within the framework of the projection theory of collective motion, a microscopic description of the rotational energy with band-mixing is formulated using a method based on an inverse power perturbation expansion in a quantity related to the expectation value of the operator $J_{y}^{2}$. The reliability of the present formulation is discussed in relation to the difference between the individual wave functions obtained from the variational equations which are established before and after projection. In addition to the various familiar quantities which appear in the phenomenological energy formula, such as the moment of inertia parameter, the decoupling factor and the band-mixing matrix element for $|\Delta K|=1$, other unfamiliar quantities having the factors with peculiar phases, $(-1)^{J+1} J(J+1),(-1)^{J+3 / 2}\left(J-\frac{1}{2}\right)\left(J+\frac{1}{2}\right)\left(J+\frac{3}{2}\right),(-1)^{J+1 / 2}\left(J+\frac{1}{2}\right) J(J+1),(-1)^{J} J(J+1)(J-1)(J+2)$ and $[J(J+1)]^{2}$ are obtained. The band-mixing term for $|\Delta K|=2$ is also new. All these quantities are expressed in terms of two-body interactions and expectation values of the operator $J_{y}^{m}$, where $m$ is an integer, within the framework of particle-hole formalism. The difference between the moment of inertia of an even-even and a neighbouring even-odd nucleus, as well as the effect of band-mixing on the moment of inertia are studied. All results are put into the forms so as to facilitate comparisons with the corresponding phenomenological terms and also for further application.
\end{abstract}

1. Introduction. - The purpose of the present paper is to present a general microscopic description of rotational bands and their mutual coupling for strongly deformed nuclei within the framework of the projection method. While the results are not all new, the present approach, which is neither a technical method of numerical calculation nor a sophisticated formal theory, provides in a systematic way a tentative microscopic interpretation of the phenomenological model description of the rotational energy and band-coupling.

The Peierls-Yoccoz theory [1-3] is a method of incorporating nuclear collective motions within a shell-model description regarding the shell-model wave function as a trial function in a variational procedure. One purely quantum mechanical approach for describing the nuclear rotation is that of projecting the deformed Hartree-Fock trial function onto the subspace of angular momentum eigenstates. This model has been widely applied [4-10] to the study of rotational properties of many nuclei.

However, the original Peierls-Yoccoz projection method has the defect of leading to an incorrect nuclear mass. Peierls and Thouless [11] subsequently 
proposed a double projection method yielding wave functions satisfying both translational and Galilean invariances and thus giving the correct total mass. Nevertheless, the application of the double projection method to the rotational problem is very cumbersome and further developments of the Peierls-Thouless theory for numerical application have not been pursued to date. Rouhaninejad and Yoccoz [12] and Zeh [13] have then shown that, when the variational principle is properly applied so as to minimize the energy of the projected wave function, the total mass for the centre of mass motion comes out correctly. The formulation from the variational procedure after projection leads, however, to very complicated equations and the relevant approximate way of handling this approach has been fully discussed by Kamlah [14], Beck, Ring, Islam and Mang [15], Onishi [16] and Villars and Schmeing-Rogerson [17]. If one confines oneself only to numerical calculations of the rotational problem, the variation of intrinsic functions after projection can be performed without difficulty provided that the model space is not so large. Thus, it has been shown that the numerical calculations in this line yielded much improved wave functions for mildly deformed nuclei [18]. It is noted that the high spin states have also been studied [19] in the framework of the microscopic variational approach. For highly deformed nuclei, however, it does not appear to matter much whether one performs the variation before or after projection [20], except probably for high spin states. In this sense, a comparison [21] between these two methods of variation used for the first three states of ${ }^{20} \mathrm{Ne}$ is very instructive. Kelson [6] has already pointed out that very little difference occurs in the intrinsic wave functions for different $J$ values of the states in ${ }^{16} \mathrm{O}$, obtained from the variation before or after projection. Indeed, it can be shown that when all quantities in the equation derived from the variational procedure after projection are expanded in powers of the quantity, $\Gamma=2 /\left\langle J_{y}^{2}\right\rangle$, then the lowest order equation is nothing but the ordinary Hartree-Fock equation and that the solutions of higher order equations can be considered as corrections to the lowest order solutions. It has been further shown [12] that as long as one keeps the constraint of unique generating function having $K=0$, the PeierlsYoccoz moment of inertia is valid. However if one takes into consideration band-mixing with $K=1$, this will be modified. Therefore, when the value of $\Gamma$ is small enough, which is the case for well deformed nuclei such as the sd-shell nuclei, it would be enough to consider only the lowest order or at most up to first order expansions.

The overlap integrals in the projection theory are generally small for nuclei of sizable deformation, except in the region of Euler angle $\beta=0$ and also in the vicinity of $\beta=\pi$. It thus turns out that the expansion of the integrands in both overlap and energy integrals in powers of $\beta$ yields [3] in first order in $\beta^{2}$, the energy spectrum formula analogous to the energy formula of the Bohr-Mottelson model [22]. A simple way of investigating the two band-mixing in the projection method has already been discussed, for example, by Verhaar [23] and by Gunye and Warke [8]. They have both employed the method of writing the $K$-mixed wave function as a sum of two $K$-pure Peierls-Yoccoz projected wave functions with mixing amplitudes. The latter authors used HartreeFock projected wave functions, whereas the former author contented himself with a pure formal approach based on both the possibility of partially expanding the overlap and energy integrals in powers of $\beta$ and using the single-particle approximation. Petry [24] has also investigated the two-band coupling problem within the framework of the Peierls-Yoccoz theory, but this study considered only the very limited case of two $K=\frac{3}{2}$ and $K=\frac{5}{2}$ bands and the expansion of the matrix elements in the vicinity of $\beta=\pi$ was completely ignored. It is thus believed that a systematic and general microscopic investigation of such band-coupling deserves further exploration.

In the present work we show a method of developing the matrix equation in the projection procedure so as to expose explicitly the rotational ingredients involved and subsequently formulate a general theory of rotational band-mixing within a microscopic basis. The discussion on the difference between the individual wave functions obtained from the variational equations established before and after projection takes also part in the present study. In order to gain an insight into the physical meaning of the results, comparisons with the Bohr-Mottelson model are made where the occasion arises. In section 2 , the expansion of the overlap and energy integrals in powers of Euler angle $\beta$ is discussed. In the following section, the expansion of these integrals in inverse powers of a quantity (designated by $X$ ), related to the width of the curve representing the matrix element $\left\langle K\left|\exp \left(-i \beta J_{y}\right)\right| K\right\rangle$, is given. In section 4 , the energy spectra are finally expanded in inverse powers of $X$, using a perturbation expansion. The correspondence between the present description and the Bohr-Mottelson model is discussed. In the last section the reliability of the present formulation is first examined in relation to generalized Hartree-Fock type equations obtained from the more relevant projection method. The rest of the section is then devoted to detailed calculations of various quantities, obtained in the preceding sections, in terms of two-body interactions and expectation values of the operator $J_{y}^{m}$, where $m$ is an integer, within the framework of the particle-hole formalism.

2. Expansion in Euler angle powers. - Let $|n K\rangle$ je the intrinsic state of quantum number $K$ which is the eigenvalue of $J_{z}$, and of additional quantum number $n$ needed to specify the intrinsic state. The $|n K\rangle$ will as a rule be a Slater determinant constructed 
with single-particle orbits. We confine ourselves here to axially symmetric Slater determinants and shall not take into consideration more released symmetric conditions.

Since the matrix element of the operator $\exp \left(-i \beta J_{y}\right)$ between the intrinsic states $|n K\rangle$ is generally small except for $\beta=0$, one uses often the approximation

$\left\langle n K\left|\exp \left(-i \beta J_{y}\right)\right| n K\right\rangle \simeq \exp \left(-\frac{\beta^{2}}{2}\left\langle J_{y}^{2}\right\rangle\right)$.

Actually, this is too rough and an improved estimate can be found using a correction factor which multiplies the Gaussian function on the right hand side of equation (1). It is noted that the operator $J_{y}$ is the sum of the individual operator $j_{y}$ over all nucleons. Verhaar [23] and Villars [4] have shown that the matrix element in equation (1) is also sizable in the neighbourhood of $\beta=\pi$ and its magnitude varies according to the values of $K$. This implies that the matrix elements of the operators $\exp \left(-i \beta J_{y}\right)$ and $H \cdot \exp \left(-i \beta J_{y}\right)$ between $|n K\rangle$ and $\left|n^{\prime} K^{\prime}\right\rangle$ may be expanded as

$$
\begin{aligned}
\left\langle n K\left|\exp \left(-i \beta J_{y}\right)\right| n^{\prime} K^{\prime}\right\rangle & = \\
& =\exp \left(-X x^{2}\right) \sum_{m=0}^{\infty} a_{K K^{\prime}}^{(m)} \cdot x^{m},
\end{aligned}
$$

and

$$
\begin{aligned}
& \left\langle n K\left|H \cdot \exp \left(-i \beta J_{y}\right)\right| n^{\prime} K^{\prime}\right\rangle= \\
& \quad=\exp \left(-X x^{2}\right) \sum_{m=0}^{\infty} \varepsilon_{K^{\prime}}^{(m)} \cdot x^{m},
\end{aligned}
$$

where $x=\frac{1}{2} \beta$ when expanding in the neighbourhood of $\beta=0$ and $x=\frac{1}{2} \beta^{\prime}$ with $\beta^{\prime}=\pi-\beta$ for the expansion around $\beta=\pi$. The expansion coefficients $a_{K^{\prime}}^{(m) \pm}$ and $\varepsilon_{K^{\prime} K^{\prime}}^{(m) \pm}$ in which the plus and minus signs stand for the expansion near $\beta=0$ and $\beta=\pi$, respectively, are then to be determined. The signification of the developments (2) and (3) as well as the meaning of the quantity $X$ can be understood by considering the expression (2), for example, for the case $n=n^{\prime}$ and $K=K^{\prime}$. Thus, when we put $X=2\left\langle J_{y}^{2}\right\rangle$ in equation (1), all terms in the summation on the right hand side of equation (2) amount to correction factors having various degrees of approximation to $\exp \left(-\frac{1}{2} \beta^{2}\left\langle J_{y}^{2}\right\rangle\right)$.

It can be shown that the quantity $X$ is related, in first order in $x^{2}$ of the expansion (2), to the expectation value of the operator $J_{y}$ by

$$
X=X_{n K}+a_{K K}^{(2)+},
$$

with

$$
X_{n K}=2\left\langle n K\left|J_{y}^{2}\right| n K\right\rangle .
$$

Equations (4) and (5) imply that the quantity $X$ may be considered as a mean value of the expectation value $X_{n K}$ averaged over the rotational bands under consideration. It is quite important to remark that the quantity $X_{n K}$ has an order of 20 or more for strongly deformed nuclei and its largeness insures quick convergences of various expansions we performe in the present formulation.

The expansion coefficients $a_{K K^{\prime}}^{(m)}$ and $\varepsilon_{K_{K^{\prime}}^{\prime}}^{(m)}$ in equation (2) and (3) may now be determined by expanding the exponential function $\exp \left(-i \beta J_{y}\right)$ on the left hand sides and then picking the same order terms of $x$ on the both sides of the equations. We thus obtain the relations :

$$
\begin{aligned}
& a_{K K^{\prime}}^{(0)+}=\delta_{n n^{\prime}} \delta_{K K^{\prime}} \\
& a_{K K^{\prime}}^{(0)-}=r \delta_{n n^{\prime}} \delta_{K K^{\prime}} \delta_{K 0}, \\
& a_{K^{\prime}}^{(1) \pm}=\mp 2\left\langle n K\left|i J_{y}\right| n^{\prime} \pm K^{\prime}\right\rangle \\
& a_{K K^{\prime}}^{(2) \pm}=X a_{K K^{\prime}}^{(0) \pm}-2\left\langle n K\left|J_{y}^{2}\right| n^{\prime} \pm K^{\prime}\right\rangle, \\
& a_{K K^{\prime}}^{(3) \pm}=X a_{K^{\prime}}^{(1) \pm} \pm \frac{4}{3}\left\langle n K\left|i J_{y}^{3}\right| n^{\prime} \pm K^{\prime}\right\rangle, \quad \text { etc... }
\end{aligned}
$$

and

$$
\begin{aligned}
& \varepsilon_{\mathbf{K K}^{\prime}}^{(0)+}=\left\langle n K|H| n^{\prime} K^{\prime}\right\rangle \delta_{n n^{\prime}} \delta_{K K^{\prime}}, \\
& \varepsilon_{\mathbf{K K}^{\prime}}^{(0)-}=\left\langle n K|H| n^{\prime}-K^{\prime}\right\rangle=r \varepsilon_{K^{\prime}}^{(0)+}, \quad \text { etc... }
\end{aligned}
$$

The higher order $\varepsilon_{\mathbf{K K}^{\prime}}^{(\boldsymbol{m}) \pm}$ can be obtained by replacing $J_{y}^{m}$ by $H J_{y}^{m}$ in Equations (6). In deriving the results (6) and (7), use was made of the definition of the timereversed state

$$
|n-K\rangle=\exp \left(-i \pi J_{y}\right)|n K\rangle,
$$

and

$$
\exp \left(-i \pi J_{y}\right)|n-K\rangle= \pm|n K\rangle,
$$

where the plus or minus signs depend on whether $K$ is integer or half-integer. In the case where $K=0$, we have

$$
\exp \left(-i \pi J_{y}\right)|n K=0\rangle=r|n K=0\rangle,
$$

with $r=+1$ for the even-parity band and $r=-1$ for the odd-parity band. In Appendix A, some higher order coefficients $a_{K^{\prime} K^{\prime}}^{(m)}$ and $\varepsilon_{K_{K}^{\prime}}^{(m) \pm}$ are shown.

By referring to the properties of the operators $J_{y}^{m}$ with $m=0,1, \ldots$, some important selection rules can be established from equations (6) and (7). We see thus

$$
\begin{array}{llll}
a_{K K^{\prime}}^{(2 m+)} & \text { and } & \varepsilon_{K^{\prime}}^{(2 m)+} & \text { vanish except for } K=K^{\prime} \pm 2 l ; \\
a_{K K^{\prime}}^{(2 m+1)+} & \text { and } & \varepsilon_{K K^{\prime}}^{(2 m+1)+} & \text { vanish except for } K=K^{\prime} \pm(2 l+1) \\
a_{K K^{\prime}}^{(2 m)-} & \text { and } & \varepsilon_{K K^{\prime}}^{(2 m)-} & \text { vanish except for } K+K^{\prime}=2 l ; \\
a_{K K^{\prime}}^{(2 m+1)-} & \text { and } & \varepsilon_{K K^{\prime}}^{(2 m+1)-} & \text { vanish except for } K+K^{\prime}=2 l+1,
\end{array}
$$

where $l=0,1, \ldots, m$. 
The Wigner rotation matrices $d_{K K^{\prime}}^{J}(\beta)$ and $d_{K K^{\prime}}^{J}\left(\beta^{\prime}=\pi-\beta\right)$ can also be expanded in powers of $x=\frac{1}{2} \beta$ and $x=\frac{1}{2} \beta^{\prime}$, respectively, using the relations, $d_{K K^{\prime}}^{J}(\beta)=(-1)^{K^{K}-K^{\prime}} d_{K^{\prime} K}^{J}(\beta)$ and $d_{K^{\prime}}^{J}\left(\pi-\beta^{\prime}\right)=$ $(-1)^{J+K} d_{K,-K^{\prime}}^{J}\left(\beta^{\prime}\right)$ as

$$
\begin{aligned}
d_{K K^{\prime}}^{J}(\beta)=(-1)^{K-K^{\prime}} & \left\{\frac{(J+K) !\left(J-K^{\prime}\right) !}{(J-K) !\left(J+K^{\prime}\right) !}\right\}^{1 / 2} \times \\
& \times \frac{x^{K-K^{\prime}}}{\left(K-K^{\prime}\right) !} \sum_{m} \gamma_{K K^{\prime}}^{(2 m)} x^{2 m},
\end{aligned}
$$

where

$$
\begin{aligned}
& \gamma_{K_{K^{\prime}}}^{(0)}=1, \\
& \gamma_{K K^{\prime}}^{(2)}=\frac{-1}{K-K^{\prime}+1}\left\{J(J+1)-K K^{\prime}-\right. \\
& \left.\quad-\frac{1}{3}\left(K-K^{\prime}\right)\left(K-K^{\prime}+1\right)\right\}, \text { etc... }
\end{aligned}
$$

with $K \geqslant K^{\prime} \geqslant 0$.

Some higher order factors $\gamma_{K K^{\prime}}^{(2 m)}$ which were used in the present description are given in Appendix B. The lower order results of the expansion of $d_{K K^{\prime}}^{J}(\beta)$ for the case with $K=K^{\prime}$ are already known $[4,22,23]$. In view of the development (11), we can put

and

$$
d_{K K^{\prime}}^{J}(\beta)=\sum_{m} \alpha_{K K^{\prime}}^{(m)+} x^{m},
$$

$$
d_{K K^{\prime}}^{J}\left(\beta^{\prime}\right)=\sum_{m} \alpha_{K K^{\prime}}^{(m)-} x^{m} .
$$

The expansion coefficients $\alpha_{K K^{\prime}}^{(m)}$ can be obtained using the properties of the Wigner rotation matrix in relation to equation (11). These coefficients are widely used throughout the present study, of which two coefficients $\alpha_{K K}^{(1)-} \delta_{K \frac{1}{2}}=-(-1)^{J+\frac{1}{2}}\left(J+\frac{1}{2}\right)$ and $\alpha_{K K}^{(2)+}=$ $-\left\{J(J+1)-K^{2}\right\}$ are well known but the higher order coefficients are not familiar (see Appendix B). An important fact is that the coefficients $\alpha_{K K^{\prime}}^{(m) \pm}$ follow the similar selection rules to those for $a_{K K^{\prime}}^{(m) \pm}$ and $\varepsilon_{K K^{\prime}}^{(m) \pm}$.

3. Perturbation expansion in inverse powers of $X$. The fact that the quantity $X$ is large can be ensure the convergence of perturbative series, provided that the expansion could be performed in inverse powers of $X$. When we substitute the various results of expansions we have made so far, into the overlap and energy integrals, we see immediately that the only factor which contains $X$ is the exponential function $\exp \left(-X x^{2}\right)$ and that the power of $x$ in the integrals is odd. Indeed, the integration of the overlap integral over $\beta$ can be transformed into the integration over the variable $x$, and we get

$$
\begin{aligned}
& N_{K^{\prime} K^{\prime}}^{J}=\int_{0}^{\pi} \sin \beta d \beta d_{K^{\prime}}^{J}(\beta) \times \\
& \times\left\langle n K\left|\exp \left(-i \beta J_{y}\right)\right| n^{\prime} K^{\prime}\right\rangle \\
& =4 \sum_{p q r}(-1)^{p} \frac{2^{p}}{(2 p+1) !}\left\{\alpha_{K^{\prime} K^{\prime}}^{(q)} a_{K^{\prime} K^{\prime}}^{(r)+}+\right. \\
& \left.+\alpha_{K K^{\prime}}^{(q)-} a_{K^{\prime} K^{\prime}}^{(r)}\right\} I_{p q r},
\end{aligned}
$$

where

$$
\begin{aligned}
& I_{p q r}= \frac{(p+s) !}{2 X^{p+s+1}}\left\{1-\exp \left(-\frac{\pi^{2}}{16} X\right) \times\right. \\
&\left.\times \sum_{k=0}^{p+s} \frac{1}{(p+s-k) !}\left(\frac{\pi^{2}}{16} X\right)^{p+s-k}\right\},
\end{aligned}
$$

with $s=\frac{1}{2}(q+r)$, which are integers because $q$ and $r$ are both even or both odd. Since the quantity $X$ is sufficiently large, the main contribution comes from the first few terms of the summation on the right hand side of equation (14), whether $p+s$ is small or large. In view of this fact, the overlap integral $N_{K K^{\prime}}^{J}$ can now be written in inverse powers of $X$ as

$$
N_{K K^{\prime}}^{J}=\frac{2}{X}\left\{n_{K K^{\prime}}^{(0)}+\frac{1}{X} n_{K K^{\prime}}^{(1)}+\frac{1}{X^{2}} n_{K K^{\prime}}^{(2)}+\cdots\right\},
$$

with

$$
\begin{aligned}
n_{K K^{\prime}}^{(m)}= & m ! \sum_{p=0}^{m}(-1)^{m-p} \frac{2^{2 m-2 p}}{(2 m-2 p+1) !} \times \\
& \times \sum_{q=0}^{2 p}\left\{\alpha_{K K^{\prime}}^{(q)+} a_{K K^{\prime}}^{(2 p-q)+}+\alpha_{K K^{\prime}}^{(q)-} a_{K K^{\prime}}^{(2 p-q)-}\right\} .
\end{aligned}
$$

These matrices $n_{K K^{\prime}}^{(m)}$ can in turn be described in terms of known matrix elements by means of the relations (6), (12) and (13).

The argument used for deriving the result (16) can equally be employed for the energy integral in order to expand it in inverse powers of $X$. Thus

$$
\begin{aligned}
\mathrm{H}_{K K^{\prime}}^{J}= & \int_{0}^{\pi} \sin \beta d \beta d_{K K^{\prime}}^{J}(\beta) \times \\
& \times\left\langle n K\left|H \cdot \exp \left(-i \beta J_{y}\right)\right| n^{\prime} K^{\prime}\right\rangle \\
= & \frac{2}{X}\left\{h_{K K^{\prime}}^{(0)}+\frac{1}{X} h_{K K^{\prime}}^{(1)}+\frac{1}{X^{2}} h_{K K^{\prime}}^{(2)}+\cdots\right\} \cdot
\end{aligned}
$$

The matrices $h_{K K^{\prime}}^{(m)}$ have the same forms as those of $n_{K K^{\prime}}^{(m)}$, except for $a_{K K^{\prime}}^{ \pm}$which are to be replaced by $\varepsilon_{K K^{\prime}}^{ \pm}$, and these matrices can be subsequently expressed in terms of known matrix elements by means of the formulas (7), (12) and (13). Thus, for example, $h_{K K^{\prime}}^{(0)}$ $=\left\langle n K|H| n^{\prime} K^{\prime}\right\rangle n_{K K^{\prime}}^{(0)}$.

The rotational energy of the state having spin $J$ and belonging to band $K$ is given by

$$
E_{\mathrm{K}}^{J}=\frac{\left[H_{\mathrm{KK}}^{J}\right]}{\left[N_{\mathrm{KK}}^{J}\right]} .
$$

If we want to incorporate the perturbative effects arising from the presence of other rotational bands, it is necessary to consider both the diagonal matrix elements and the off-diagonal ones of the matrix constructed from the ratio of the matrix $[H]$ to the matrix $[N]$. Here we distinguish the difference between the ratio matrix and the ratio of the two matrices. The immediate aim is now to expand such a ratio matrix in inverse powers of $X$. However, some care is 
needed in constructing the ratio matrix owing to the antisymmetric character of the product matrix. Indeed, the product matrices $[N]^{-1}[H]$ and $[H][N]^{-1}$ are not symmetric with each other. Since the matrix $[N]$ is not singular, we have an inverse matrix $[N]^{-1}$ which is symmetric if $[N]$ is symmetric. Because the matrix $[N]$ is defined to be positive, there exists a matrix $[N]^{-1 / 2}$ which is also symmetric. As a consequence, we construct the ratio matrix as

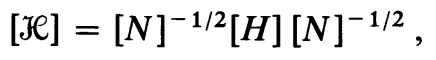

which is now symmetric and possesses the same eigenvalue as of equation (19). When substitutions of equations (16) and (18) are made into equations (20), we may finally expand the energy matrix [HC] in inverse powers of $X$ as

$$
[\mathcal{H}]=\left[H^{(0)}\right]+\frac{1}{X}\left[H^{(1)}\right]+\frac{1}{X^{2}}\left[H^{(2)}\right]+\cdots
$$

with

$$
\begin{aligned}
& H^{(0)}=n^{(0)} h^{(0)}, \\
& H^{(1)}=n^{(0)}\left\{h^{(1)}-\frac{1}{2} n^{(0)}\left[n^{(1)} h^{(0)}+h^{(0)} n^{(1)}\right]\right\},
\end{aligned}
$$

where $n^{(0)}$ is the inverse of $n^{(0)}$. The matrices $n^{(m)}$ and $h^{(m)}$ are defined in equations (16), (17) and (18) and here we have for simplicity omitted the matrix symbol [ ] in equations (21.1) and (21.2). The higher order matrices $H^{(m)}$ up to $m=4$ are given in Appendix C. It is remarked that when $m=0$, equation (17) reduces to the identity matrix for even-odd nuclei, and becomes diagonal for even-even nuclei with $n_{K K^{\prime}}^{(0)}=\delta_{K K^{\prime}}$ for non zero integer values of $K$ and with $n_{K K^{\prime}}^{(0)}=\left[1+r(-1)^{J} \delta_{K 0}\right] \delta_{K K^{\prime}}$ for $K=K^{\prime}=0$. The matrix $n^{(0)}$ is diagonal and non singular, and the matrix $H^{(0)}$ is also diagonal in the present representation.

4. Energy level. - In the presence of several rotational bands, the perturbed energy level of spin $J$ belonging to the rotational band $K_{i}$ is now expressed in inverse powers of $X$ as

$$
E_{K_{i}}^{J}=E_{K_{i}}^{(0)}+\frac{1}{X} E_{K_{i}}^{(1)}+\frac{1}{X^{2}} E_{K_{i}}^{(2)}+\frac{1}{X^{3}} E_{K_{i}}^{(3)}+\cdots,
$$

with

$$
E_{K_{i}}^{J}=\left[\mathcal{H}_{K_{i} K_{i}}^{J}\right],
$$

$$
\begin{aligned}
E_{K_{i}}^{(0)} & =H_{i i}^{(0)}, \\
E_{K_{i}}^{(1)} & =H_{i i}^{(1)}, \\
E_{K_{i}}^{(2)} & =H_{i i}^{(2)}+\sum_{j \neq i} \frac{H_{i j}^{(1)} H_{j i}^{(1)}}{E_{i}-E_{j}}, \\
E_{K_{i}}^{(3)} & =H_{i i}^{(3)}+\sum_{j \neq i} \frac{H_{i j}^{(1)} H_{j i}^{(2)}+H_{i j}^{(2)} H_{j i}^{(1)}}{E_{i}-E_{j}}+ \\
& +\sum_{j, k \neq i} \frac{H_{i j}^{(1)} H_{j k}^{(1)} H_{k i}^{(1)}}{\left(E_{i}-E_{j}\right)\left(E_{i}-E_{k}\right)}-H_{i i}^{(1)} \sum_{j \neq i} \frac{H_{i j}^{(1)} H_{j i}^{(1)}}{\left(E_{i}-E_{j}\right)^{2}} .
\end{aligned}
$$

Here, the notation $H_{i j}^{(m)}$ stands for the matrix element between the states $K_{i}$ and $K_{j}$ of order $m$ in the expansion of the matrix [He] of equation (21). We, see that $E_{K_{i}}^{(m)}$ with $m \geqslant 1$ represent correction terms to unperturbed energy $E_{K_{i}}^{(0)}$. It is worthwhile at this step to mention that, because of the expansions (2) and (3) we made at the beginning, the usual expanding factors like $\int \sin \beta d \beta \beta^{m} N_{0}(\beta) / \int \sin \beta d \beta N_{0}(\beta)$ which stroll through the formulation in other studies of the projection method do not appear in the present work. The first order correction $E_{K_{i}}^{(1)}$ has only diagonal matrix element but the higher order corrections have off-diagonal matrix elements as well as the diagonal ones. It is noticed that the necessity of having the factor $\left[J(J+1)-K^{2}\right]^{2}$ as well as the bandmixing term for $|\Delta K|=2$ urges us to the evaluation of the perturbation expansion up to fourth order in $1 / X$. Generally, perturbation theory can be applied to the band-mixing problem with confidence when the off-diagonal matrix elements are small compared to the difference between the diagonal matrix elements for different $K$. In other words, the perturbation expansion is valid mostly for the case in which the heads of the bands in consideration are located sufficiently far from each other and/or the off-diagonal matrix elements are small. Naturally, the applicability of the present approach depends to a great extent on the value of $X$.

4.1 FIRST ORDER ENERGY CORRECTION. - In the first order energy correction, we have only the diagonal matrix element which is written in terms of $n^{(m)}$ and $h^{(m)}$ having $m=0$ and 1 .

$$
\begin{aligned}
E_{K_{i}}^{(1)} & =n_{i i}^{(0)}\left\{h_{i i}^{(1)}-\frac{1}{2} n_{i i}^{(0)} \sum_{k}\left[n_{i k}^{(1)} h_{k i}^{(0)}+h_{i k}^{(0)} n_{k i}^{(1)}\right]\right\} \\
& =n_{i i}^{(0)}\left\{h_{i i}^{(1)}-E_{K_{i}}^{(0)} n_{i i}^{(1)}\right\} .
\end{aligned}
$$

When use made of the definitions of matrices $n^{(1)}$ and $h^{(1)}$ in equations (16) and (18), then equation (24) reduces to

$$
\begin{aligned}
E_{K_{i}}^{(1)}=- & 2\left\{\left\langle n_{i} K_{i}\left|H J_{y}^{2}\right| n_{i} K_{i}\right\rangle-\left\langle n_{i} K_{i}|H| n_{i} K_{i}\right\rangle\left\langle n_{i} K_{i}\left|J_{y}^{2}\right| n_{i} K_{i}\right\rangle\right\}- \\
& -2 \delta_{K_{i} \frac{1}{2}}(-1)^{J+\frac{1}{2}}\left(J+\frac{1}{2}\right)\left\{\left\langle n_{i} K_{i}\left|H i J_{y}\right| n_{i}-K_{i}\right\rangle-\left\langle n_{i} K_{i}|H| n_{i} K_{i}\right\rangle\left\langle n_{i} K_{i}\left|i J_{y}\right| n_{i}-K_{i}\right\rangle\right\} .
\end{aligned}
$$


It is remarked that, except for the case of $K_{i}=\frac{1}{2}$, the first order correction to $E_{K_{i}}^{(0)}$ is independent of spin $J$ and this term multiplied by $1 / X$ is known $[12,13,15,22]$ as $-\left\langle J_{y}^{2}\right\rangle / \Theta$, where $\Theta$ is the moment of inertia of PeierlsYoccoz. The terms in the second bracket is related to the decoupling parameter of the Bohr-Mottelson model.

4.2 SECOND ORDER ENERGY CORRECTION. - With the aid of the matrices $h^{(2)}$ and $n^{(2)}$ in addition to the matrices $h^{(1)}$ and $n^{(1)}$, the second order energy correction terms may be written as

$$
E_{\mathbf{K}_{i}}^{(2)}=n_{i i}^{(0)}\left\{h_{i i}^{(2)}-E_{K_{i}}^{(0)} n_{i i}^{(2)}-E_{K_{i}}^{(1)} n_{i i}^{(1)}+n_{i i}^{(0)} \sum_{l \neq i} \frac{\left[h_{i l}^{(1)}-E_{i l}^{(0)} n_{i l}^{(1)}\right]\left[h_{l i}^{(1)}-E_{i l}^{(0)} n_{l i}^{(1)}\right]}{E_{K_{i}}^{(0)}-E_{K_{l}}^{(0)}}\right\},
$$

with $E_{i l}^{(0)}=\frac{1}{2}\left(E_{K_{i}}^{(0)}+E_{K_{l}}^{(0)}\right)$.

This formula can be subsequently expressed in terms of known and familiar matrix elements as in equation (25). To simplify lengthy expressions, we introduce the abbreviated notations,

$$
\begin{aligned}
\left\langle J_{y}^{m}\right\rangle_{i \pm k} & =\left\langle n_{i} K_{i}\left|J_{y}^{m}\right| n_{k} \pm K_{k}\right\rangle, \\
\left\langle H J_{y}^{m}\right\rangle_{i \pm k} & \left\langle\left\langle n_{i} K_{i}\left|H J_{y}^{m}\right| n_{k} \pm K_{k}\right\rangle-\frac{1}{2}\left\{\left\langle n_{i} K_{i}|H| n_{i} K_{i}\right\rangle+\right.\right. \\
& \left.\quad+\left\langle n_{k} K_{k}|H| n_{k} K_{k}\right\rangle\right\}\left\langle n_{i} K_{i}\left|J_{y}^{m}\right| n_{k} \pm K_{k}\right\rangle .
\end{aligned}
$$

With these notations we have

$$
\begin{aligned}
E_{K_{i}}^{(2)}= & \frac{4}{3}\left\langle H J_{y}^{4}\right\rangle_{i i}-2\left\{X+2\left\langle J_{y}^{2}\right\rangle_{i i}-\frac{2}{3}+2 \delta_{K_{i} \frac{1}{2}}(-1)^{J+\frac{1}{2}}\left(J+\frac{1}{2}\right)\left\langle i J_{y}\right\rangle_{i-i}\right\} \times \\
& \times\left\{\left\langle H J_{y}^{2}\right\rangle_{i i}+\delta_{K_{i} \frac{1}{2}}(-1)^{J+\frac{1}{2}}\left(J+\frac{1}{2}\right)\left\langle H i J_{y}\right\rangle_{i-i}\right\} \\
& -\frac{1}{3} \delta_{K_{i} \frac{1}{2}}(-1)^{J+\frac{1}{2}}\left(J+\frac{1}{2}\right)\left\langle H i J_{y}\right\rangle_{i-i}+2\left[J(J+1)-K_{i}^{2}\right]\left\langle H J_{y}^{2}\right\rangle_{i i} \\
& -2 \delta_{K_{i} 1}(-1)^{J+1} J(J+1)\left\langle H J_{y}^{2}\right\rangle_{i-i}+\frac{8}{3} \delta_{K_{i} \frac{1}{2}}(-1)^{J+\frac{1}{2}}\left\langle H i J_{y}^{3}\right\rangle_{i-i} \\
& +4 \sum_{l \neq i} \frac{1}{E_{K_{i}}^{(0)}-E_{K_{l}}^{(0)}}\left|M_{i l}^{(2)}\right|^{2}
\end{aligned}
$$

with

$$
\begin{aligned}
M_{i l}^{(2)}= \pm \delta_{K_{i}, K_{l} \pm 1} \sqrt{J(J+1)-K_{i} K_{l}} & \left.<H i J_{y}\right\rangle_{i l}+\left\{ \pm \delta_{K_{i}\left(\begin{array}{l}
1 \\
1
\end{array}\right)} \delta_{K_{l}\left(\frac{1}{0}\right)} \times\right. \\
& \left.\left.\times(-1)^{J+1} \sqrt{J(J+1)}-\delta_{K_{i} K_{l}} \delta_{K_{i} \frac{1}{2}}(-1)^{J+\frac{1}{2}}\left(J+\frac{1}{2}\right)\right\}\left\langle H i J_{y}\right\rangle_{i-l}\right\}
\end{aligned}
$$

Here we find, in addition to the famous factor $J(J+1)-K_{i}^{2}$, the band-mixing element for $|\Delta K|=1$. It is interesting to note that the present study gives a particular term which couples two $K_{i}=\frac{1}{2}$ bands having different structures. Correspondences between the terms from the Bohr-Mottelson and the present result are discussed in detail at the end of this section.

4.3 THIRD ORDER ENERGY CORRECTION. - Instead of writing down complete and lengthy expression of the third order energy correction, we will confine ourselves here to some particular results contained in $E_{\mathbf{K}_{i}}^{(3)}$, in order to understand the various contributions coming from the third order correction. The correction $E_{K_{i}}^{(3)}$ can be classified into terms which are independent of spin $J$, those terms which contain the factor $J(J+1)-K_{i}^{2}$, the terms which couple the different bands and finally those terms which are specific only to $K_{i}=\frac{1}{2}, 1$ and $\frac{3}{2}$. One surprising result of the present description of the rotational energy is that even the third order energy correction in $1 / X$ does not yield the terms containing the factor $\left[J(J+1)-K_{i}^{2}\right]^{2}$ and it is necessary to calculate the fourth order correction in order to find the square of $J(J+1)$ as well as the band-mixing term for $|\Delta K|=2$. Actually, $E_{K_{i}}^{(3)}$ contains the elements like $\alpha_{K_{i} K_{i}}^{(4)+}$ and $\left(\alpha_{K_{i} K_{i}}^{(2)+}\right)^{2}$ which are respectively proportional to $\left[J(J+1)-K_{i}^{2}\right]^{2}$ but the sum $8 \alpha_{K_{i} K_{i}}^{(4)+}-2\left(\alpha_{K_{i} K_{i}}^{(2)+}\right)^{2}$ which appears in $E_{K_{i}}^{(3)}$ vanishes and there is thus no term having the square of $J(J+1)$ and this is the reason why several studies in the past have predicted this term but have never formulated the exact expression. Instead, we have the factor $J(J+1)-K_{i}^{2}$ multiplied by various matrix elements, viz :

$$
\left\{J(J+1)-K_{i}^{2}\right\}\left\{-\frac{8}{3}\left\langle H J_{y}^{4}\right\rangle_{i i}+\left(4 X+8\left\langle J_{y}^{2}\right\rangle_{i i}-\frac{4}{3}\right)\left\langle H J_{y}^{2}\right\rangle_{i i}\right\} .
$$


There are other terms which are proportional to $(-1)^{J+\frac{1}{2}}\left(J+\frac{1}{2}\right) J(J+1)$ and which are specific to $K_{i}=\frac{1}{2}$ only, namely,

$$
\begin{aligned}
4 \delta_{K_{i} \frac{1}{2}}(-1)^{J+\frac{1}{2}}\left(J+\frac{1}{2}\right) J(J+1)\left\{-\frac{1}{3}\left\langle H i J_{y}^{3}\right\rangle_{i-i}\right. & +\left\langle i J_{y}\right\rangle_{i-i}\left\langle H J_{y}^{2}\right\rangle_{i i}+ \\
& \left.+\left\langle J_{y}^{2}\right\rangle_{i i}\left\langle H i J_{y}\right\rangle_{i-i}-2\left\langle i J_{y}\right\rangle_{i-i}^{2}\left\langle H i J_{y}\right\rangle_{i-i}\right\} .
\end{aligned}
$$

The quantity

$$
\frac{4}{3} \delta_{K_{i} \frac{3}{2}}(-1)^{J+\frac{3}{2}}\left(J+\frac{3}{2}\right)\left(J+\frac{1}{2}\right)\left(J-\frac{1}{2}\right)\left\langle H J_{y}^{3}\right\rangle_{i-i},
$$

is specific to $K_{i}=\frac{3}{2}$ and these terms may be compared with the terms having the same $J$ factor in the phenomenological energy formula.

The off-diagonal matrix elements such that $h_{i l}^{(2)}, h_{l i}^{(1)}$, etc... are related to band-mixing terms between $K_{i}$ and $K_{l}$, satisfying the relation $K_{i}=K_{l} \pm 1$ or $K_{i}+K_{l}=1$. It is also seen that the band-mixing terms having the matrix elements $h_{i l}^{(1)}, h_{l m}^{(1)}$ and $h_{m i}^{(1)}$ are allowed only for $K_{m}=K_{l}$ and $K_{i}=K_{l} \pm 1$ or $K_{i}+K_{l}=1$. These band-mixing terms are rather lengthy and less important than the one obtained in the second order correction so that we do not give any explicit forms of them.

4.4 FOURTH ORDER ENERGY CORRECTION. - The fourth order energy correction is composed of terms similar to those found in the third order correction : terms which are independent of spin $J$, terms with the factor $J(J+1)-K_{i}^{2}$, band-mixing terms for $K_{i}=K_{l} \pm 1$ and $K_{i}+K_{l}=1$, and those terms specific to the $K_{i}=\frac{1}{2}$, $1, \frac{3}{2}$ and 2 bands. In addition, we have the term having the square of $J(J+1)$ of the form,

$$
\left\{J(J+1)-K_{i}^{2}\right\}^{2}\left\{\frac{2}{3}\left\langle H J_{y}^{4}\right\rangle_{i i}-4\left\langle J_{y}^{2}\right\rangle_{i i}\left\langle H J_{y}^{2}\right\rangle_{i i}\right\} .
$$

There is no terms proportional to the cube of $J(J+1)$ and we would have to go to higher order corrections in order to find them.

We have three more new terms, of which one is specific to $K_{i}=2$,

$$
\frac{2}{3} \delta_{K_{i} 2}(-1)^{J}(J-1) J(J+1)(J+2)\left\langle H J_{y}^{4}\right\rangle_{i-i}
$$

and the second is specific to $K_{i}=1$,

$$
\begin{aligned}
\delta_{K_{i} 1}(-1)^{J+1} J^{2}(J+1)^{2}\left\{-\frac{2}{3}\left\langle H J_{y}^{4}\right\rangle_{i-i}+4\left\langle J_{y}^{2}\right\rangle_{i-i}\left\langle H J_{y}^{2}\right\rangle_{i i}+4\left\langle J_{y}^{2}\right\rangle_{i i}\left\langle H J_{y}^{2}\right\rangle_{i-i}-\right. \\
\left.-4(-1)^{J+1}\left\langle J_{y}^{2}\right\rangle_{i-i}\left\langle H J_{y}^{2}\right\rangle_{i-i}\right\} .
\end{aligned}
$$

The third is the band-mixing element between $K_{i}$ and $K_{l}$ satisfied by the conditions $\left|K_{i}-K_{l}\right|=2$ or $K_{i}+K_{l}=2, v i z$ :

$$
\sum_{l \neq i} \frac{1}{E_{\mathbf{K}_{i}}^{(0)}-E_{\mathbf{K}_{l}}^{(0)}}\left|M_{i l}^{(4)}\right|^{2},
$$

with

$$
\begin{aligned}
M_{i l}^{(4)}=2\left\{\delta_{K_{l}, K_{i} \pm 2} \sqrt{\left[J(J+1)-K_{i}\left(K_{i} \pm 1\right)\right]\left[J(J+1)-\left(K_{i} \pm 1\right)\left(K_{i} \pm 2\right)\right]} \times\right. \\
\\
\times\left\langle H J_{y}^{2}\right\rangle_{i l}+\left[\delta_{K_{i}\left(\frac{1}{2}\right)} \delta_{K_{l}\left(\frac{2}{2}\right)}(-1)^{J} \sqrt{J(J-1)(J+1)(J+2)}\right. \\
\\
\pm \delta_{K_{i}\left(\frac{1}{3 / 2}\right)} \delta_{\left.K_{l} l_{1 / 2}^{3 / 2}\right)}(-1)^{J+\frac{1}{2}}\left(J+\frac{1}{2}\right) \sqrt{\left(J+\frac{3}{2}\right)\left(J-\frac{1}{2}\right)} \\
\left.\left.+\delta_{K_{i} 1} \delta_{K_{l} 1}(-1)^{J+1} J(J+1)\right]\left\langle H J_{y}^{2}\right\rangle_{i-l}\right\}
\end{aligned}
$$

Rowe [25] has studied the possibility of two-band mixing for $|\Delta K|=2$ from a phenomenological point of view and concluded that the effects of this coupling are negligible for ${ }^{183} \mathrm{~W}$ nucleus. Since this mixing term is in the fourth order correction we have actually to divide the expression (35) by $X^{4}$ and the effect of the band-mixing with $|\Delta K|=2$ is thus seen to be very small.

Gathering all the results we have established so far, we are now able to write the rotational energy level for spin $J$, belonging to the band $K_{i}$ upon which other bands $K_{l}$ have the effects, in the more familiar form,

$$
E_{K_{i}}^{J}=\left\{E_{K_{i}}^{(0)}-\frac{2}{X}\left\langle H J_{y}^{2}\right\rangle_{i i}+\left[\frac{1}{X^{2}} W_{0}^{(2)}+\frac{1}{X^{3}} W_{0}^{(3)}+\frac{1}{X^{4}} W_{0}^{(4)}\right]\right\}+
$$




$$
\begin{aligned}
& +\left\{J(J+1)-K_{i}^{2}\right\}\left\{\frac{2}{X^{2}}\left\langle H J_{y}^{2}\right\rangle_{i i}+\left[\frac{1}{X^{3}} W_{J}^{(3)}+\frac{1}{X^{4}} W_{J}^{(4)}\right]\right\} \\
& +\left\{J(J+1)-K_{i}^{2}\right\}^{2}\left\{\frac{1}{X^{4}}\left[\frac{2}{3}\left\langle H J_{y}^{4}\right\rangle_{i i}-4\left\langle J_{y}^{2}\right\rangle_{i i}\left\langle H J_{y}^{2}\right\rangle_{i i}\right]\right\} \\
& +\delta_{K_{i} \frac{1}{2}}(-1)^{J+\frac{1}{2}}\left(J+\frac{1}{2}\right)\left\{-\frac{2}{X}\left\langle H i J_{y}\right\rangle_{i-i}+\left[\frac{1}{X^{2}} W_{\frac{1}{2}}^{(2)}+\frac{1}{X^{3}} W_{\frac{1}{2}}^{(3)}+\frac{1}{X^{4}} W_{\frac{1}{2}}^{(4)}\right]\right\} \\
& +\delta_{K_{i} 1}(-1)^{J+1} J(J+1)\left\{-\frac{2}{X^{2}}\left\langle H J_{y}^{2}\right\rangle_{i-i}+\left[\frac{1}{X^{3}} W_{1}^{(3)}+\frac{1}{X^{4}} W_{1}^{(4)}\right]\right\} \\
& +\delta_{K_{i} \frac{3}{2}}(-1)^{J+\frac{3}{2}}\left(J+\frac{3}{2}\right)\left(J+\frac{1}{2}\right)\left(J-\frac{1}{2}\right)\left\{\frac{1}{X^{3}} \frac{4}{3}\left\langle H i J_{y}^{3}\right\rangle_{i-i}+\frac{1}{X^{4}} W_{\frac{3}{2}}^{(4)}\right\} \\
& +\delta_{K_{i} 2}(-1)^{J}(J+2)(J+1) J(J-1)\left\{\frac{1}{X^{4}} \frac{2}{3}\left\langle H J_{y}^{4}\right\rangle_{i-i}\right\} \\
& +\sum_{l \neq i} \frac{1}{E_{K_{i}}^{(0)}-E_{K_{l}}^{(0)}}\left\{\left[\frac{4}{X^{2}}\left|M_{i l}^{(2)}\right|^{2}+\left(\frac{1}{X^{3}} W_{i l}^{(3)}+\frac{1}{X^{4}} W_{i l}^{(4)}\right)\right] \delta_{K_{i}, K_{l} \pm 1}\left(\delta_{K_{i}+K_{l}, 1}\right)\right. \\
& \left.+\frac{1}{X^{4}}\left|M_{i l}^{(4)}\right|^{2} \delta_{K_{l}, K_{i} \pm 2}\left(\delta_{K_{l}+K_{i}, 2}\right)+L_{i l}\right\}
\end{aligned}
$$

where the band-mixing matrix elements $M_{i l}^{(2)}$ and $M_{i l}^{(4)}$ are given in equations (28) and (36). Explicit forms of some of the functions designated by $W^{(m)}$, where $m$ stands for the expansion order in $X$, are shown in Appendix D. It is noticed that for the purpose of understanding the physical meaning of the quantities contained in the present description only the leading order contributions to each geometrical factor of $J$ are explicitly shown in equation (37). At this stage, it is very tentative to compare the formula (37) with the phenomenological expression given by Bohr and Mottelson [22], namely

$$
\begin{array}{rlr}
E(K, J)=E_{K}+A J(J+1) & +B J^{2}(J+1)^{2}+\cdots+ \\
& +(-1)^{J+\frac{1}{2}}\left(J+\frac{1}{2}\right)\left(A_{1}+B_{1} J(J+1)+\cdots\right), & K=\frac{1}{2} \\
& +(-1)^{J+1} J(J+1)\left(A_{2}+B_{2} J(J+1)+\cdots\right), & K=1 \\
& +(-1)^{J+\frac{3}{2}}\left(J-\frac{1}{2}\right)\left(J+\frac{1}{2}\right)\left(J+\frac{3}{2}\right)\left(A_{3}+B_{3} J(J+1)+\cdots\right), & K=\frac{3}{2} \\
& +(-1)^{J}(J-1) J(J+1)(J+2)\left(A_{4}+B_{4} J(J+1)+\cdots\right), & K=2
\end{array}
$$

or with similar forms which can be obtained by replacing $J(J+1)$ by $J(J+1)-K^{2}$. However, much care is needed whenever a direct comparison is made between two corresponding terms in these formulas, since it is not obvious that these terms carry the same weight in their respective contributions. For example, the term with the factor $\left[J(J+1)-K^{2}\right]^{2}$ in the present study appears only in the fourth order in $1 / X$ and therefore the contribution of this term to the total energy should be considered with relation to all other contributions from the fourth order correction. Moreover, equation (38) contains no band-mixing terms neither for $|\Delta K|=1$ nor for $|\Delta K|=2$, whereas the present description takes into account the effects of band-mixing without introducing the Coriolis force. Therefore, the interpretation of the factor $A_{1}$, for example, as the diagonal effect of the Coriolis forces acting in the rotating coordinate system may not be applied without caution to the corresponding matrix element $-\frac{2}{X}\left\langle H i J_{y}\right\rangle_{i-i}$ in equation (37). Various terms arising in the particle-rotor model may provide however a way of understanding the corresponding terms in equation (37).

The explicit expressions similar to the factors $B_{1}$ and $B_{2}$ in equation (37) can be obtained from the functions $W_{1 / 2}^{(2)}$ and $W_{1}^{(3)}$, and these have been already shown in equations (30) and (34). The factors $B_{3}$ and $B_{4}$ cannot be obtained in the present work as it is limited to fourth order in $1 / X$ and it would be necessary to go to higher order expansion in order to include them.

For even-even nuclei, the energy spectrum up to $1 / X^{2}$ order becomes

$$
E_{0}^{J}=E_{0}+\left(A+A^{\prime}\right) J(J+1),
$$

with

$E_{0}=\left\{E_{0}^{(0)}+\frac{1}{X^{2}} W_{0}^{(2)}\right\}-\frac{\frac{1}{2} X}{\Theta}$,

$A=\frac{1}{2 \Theta}$ 


$$
\begin{array}{r}
A^{\prime}=\frac{1}{X^{2}} \cdot \sum_{l} \frac{1}{E_{0}^{(0)}-E_{\mathbf{K}_{l}}^{(0)}} \delta_{K_{l} 1} \mid\left\langle H i J_{y}\right\rangle_{i l}+ \\
+\left.(-1)^{J}\left\langle H i J_{y}\right\rangle_{i-l}\right|^{2} .
\end{array}
$$

In deriving these formulas, use is made of the moment of inertia of Peierls-Yoccoz, $\Theta=(X / 2)^{2} /\left\langle H J_{y}^{2}\right\rangle_{i i}$. The function $A^{\prime}$ is the correction term to the inertia parameter $A$ and this comes from the mixing of the $K_{i}=0$ band with $K_{l}=1$ bands. The expression(39.3) is a concise form of the correction which has already been predicted by Yoccoz [12] but has not yet been formulated. Apart from the band-mixing term, the formula (39) is well known and this is to be compared with the more general formula of Beck et al. [15] and Villars et al. [17]. Because of the severe symmetry condition we have preserved throughout the formulation, we cannot have the terms having the expectation values of the operators other than $J_{y}^{m}$.

5. Self-consistent field. - Before pursueing further it is now necessary at this state to discuss the difference between the intrinsic wave functions obtained variationally before and after projection. For simplicity we confine ourselves here to the case of eveneven nuclei with axial symmetry and we follow closely the method of reference [12].

The variational principle applied to the state projected out of the Slater determinant constructed with individual wave functions $u$ yields

$$
\int d(\cos \beta) P_{J}(\cos \beta) N(\beta) F(u, \beta)=0,
$$

where $P_{J}$ is the Legendre polynomials and $N(\beta)$ is the matrix element of equation (2) for even-even nuclei with the intrinsic states which may now depend on $J$. The function $F(u, \beta)$, which is also a function of various energies involved, is shown explicitly in Appendix E. When all terms in this equation are expanded in powers of $X$, and further the wave function $u$ is developed in inverse powers of $X$ as

$$
u=u^{(0)}+\frac{1}{X} u^{(1)}-\frac{1}{X^{2}} u^{(2)}+\cdots
$$

we obtain then a rather complicated equation which contains different orders of $F(u, \beta)$,

$$
\begin{aligned}
& F_{0}^{(0)}\left(u^{0}\right)+\frac{1}{X}\left\{F_{0}^{(1)}\left(u^{0}, u^{1}\right)+\overline{\beta^{2}}\left[F_{2}^{(0)}\left(u^{0}\right)+\right.\right. \\
& \left.\left.\quad+\left(b_{2}-\frac{1}{4} J(J+1)\right) F_{0}^{(0)}\left(u^{0}\right)\right]\right\}+\cdots=0,
\end{aligned}
$$

where $b_{2}$ is the coefficient of the $\beta^{2}$ term in the expansion of $N(\beta)$ and the averaged $\overline{\beta^{2}}$ is $X / 4$ times the factor shown in the discussion which follows equation (23). In view of the explicit form of $F(u, \beta)$ given in Appendix $\mathrm{E}$, it is seen that the lowest order equation, $F_{0}^{(0)}\left(u^{0}\right)=0$, is nothing but the ordinary Har-
tree-Fock equation. If there exist a solution of the lowest order, then the first order equation, which becomes now $F_{0}^{(1)}\left(u^{0}, u^{1}\right)+\overline{\beta^{2}} F_{2}^{(0)}\left(u^{0}\right)=0$ and which is independent of $J$, can be solved by the matrix inversion method. Since the quantity $X$ is large for well deformed nuclei, it is believed that the consideration of the solutions of $u$ up to first order would be sufficient for the correction to the lowest order solution. This shows clearly the limit and the validity of the method which use the ordinary Hartree-Fock solutions in the projected formulas. For example, it was shown [12] that the old formula for the moment of inertia, $\Theta$, is still valid when the ordinary HartreeFock solutions are used, since the $J$-dependent correction to $u^{(0)}$ appears only in the second order in $1 / X$ and thus modify the rotational energy in the terms in $1 / X^{4}$ order. It should be, however, noticed that the argument used for the discussion of reliability of the lowest order solution in the rotational problem makes not much sense for the centre of mass problem, since the first order solution is as important as the lowest order solution in the calculation of the translational constant.

In what follows, the intrinsic state $|n K\rangle$ will be considered to be a self-consistent solutions of the Hamiltonian of strongly deformed nuclei in an axially symmetry field. It is desired that the $|n K\rangle$ is at least the first order solutions of equation (42). When the self-consistent basis stands only for the solutions from the ordinary Hartree-Fock equation, then the formulation below has to be regarded in the restricted sense.

The zero order energy in equation (22) now becomes

$$
\begin{aligned}
E_{K}^{(0)}= & \frac{1}{1+(-1)^{J} \delta_{K 0}}\{\langle n K|H| n K\rangle+ \\
& \left.+(-1)^{J}\langle n K|H| n-K\rangle \delta_{K 0}\right\} \\
= & \langle n K|H| n K\rangle,
\end{aligned}
$$

which implies even values of $J$ for $K=0$, as was expected.

Suppose now we have a set of states in which $|i\rangle=a_{i}^{+}|0\rangle$ represents a one-particle state, $|\alpha\rangle=b_{\alpha}^{+}|0\rangle$ represents a one-hole state and $|i \alpha\rangle=a_{i}^{+} b_{\alpha}^{+}|0\rangle$ represents a one-particle onehole state and so on, where $|0\rangle$ stands for the ground state of an even-even nucleus which may subsequently become the core for the nearest even-odd nuclei under consideration. Here, italic indices $i, j, k \ldots$ are associated with particle states and greek indices $\alpha, \beta, \gamma \ldots$ are associated with hole states.

The nuclear Hamiltonian is now written in the form

$$
\begin{array}{r}
H=\sum_{i} \varepsilon_{i} a_{i}^{+} a_{i}-\sum_{\alpha} \varepsilon_{\alpha} b_{\alpha}^{+} b_{\alpha}+H_{40}+H_{04}+ \\
+H_{31}+H_{13}+H_{22},
\end{array}
$$

where $\varepsilon_{i}$ and $\varepsilon_{\alpha}$ are respectively energies of the particle and hole and $H_{40}, H_{04}$ etc. have their usual meanings, 
namely, $H_{m n}$ stands for the two-body interaction term having $m$ creation and $n$ annihilation operators (particles or/and holes).

The operator $J_{y}$, written in the same particle-hole formalism, is given by

$$
\begin{aligned}
J_{y}=\sum_{i k}\langle & \left.J_{y}\right\rangle_{i k} a_{i}^{+} a_{k}+\sum_{i \alpha}\left\langle J_{y}\right\rangle_{i \alpha} a_{i}^{+} b_{\alpha}^{+}+ \\
& +\sum_{i \alpha}\left\langle J_{y}\right\rangle_{\alpha i} b_{\alpha} a_{i}-\sum_{\alpha \beta}\left\langle J_{y}\right\rangle_{\alpha \beta} b_{\beta}^{+} b_{\alpha},
\end{aligned}
$$

where $\left\langle J_{y}\right\rangle_{i \alpha}=\left\langle i\left|J_{y}\right| \alpha\right\rangle$. Similarly

$$
J_{y}^{2}=\left(J_{y}^{2}\right)_{0}+\left(J_{y}^{2}\right)_{1}+\left(J_{y}^{2}\right)_{2},
$$

where three operators on the right hand side represent a constant, one-body and two-body operators, respectively (see Appendix F). The operators $J_{y}^{3}$ and $J_{y}^{4}$, which have been used for explicit calculations of some matrix elements, can be expressed in second quantization forms. Indeed, the operator $J_{y}^{3}$ is composed of one-body, two-body and three-body operators, whilst the operator $J_{y}^{4}$ is composed of a constant, one-body, two-body, three-body and four-body operators. For actual calculations, we could equally use the product forms of these operators, namely, $J_{y}^{3}=J_{y} \times J_{y}^{2}$ and $J_{y}^{4}=J_{y}^{2} \times J_{y}^{2}$ by introducing a complete set of intermediate states between each component operator.

Since $H$ and $J_{y}^{m}$ commute each other, the matrix element (27.2) becomes

$$
\begin{aligned}
\left\langle H J_{y}^{m}\right\rangle_{i l} & =\frac{1}{2} \sum_{n^{\prime} K^{\prime}}^{\prime}\left\{\left\langle n_{i} K_{i}|H| n^{\prime} K^{\prime}\right\rangle \times\right. \\
& \times\left\langle n^{\prime} K^{\prime}\left|J_{y}^{m}\right| n_{l} K_{l}\right\rangle+\left\langle n_{i} K_{i}\left|J_{y}^{m}\right| n^{\prime} K^{\prime}\right\rangle \\
& \left.\times\left\langle n^{\prime} K^{\prime}|H| n_{l} K_{l}\right\rangle\right\},
\end{aligned}
$$

where the prime on the sigma indicates $\left(n^{\prime} K^{\prime}\right) \neq$ $\left(n_{i} K_{i}\right),\left(n_{l} K_{l}\right)$. Generally, $\sum\left|n^{\prime} K^{\prime}\right\rangle\left\langle n^{\prime} K^{\prime}\right|$ represents the sum of the complete set and satisfies the closure condition. Because of the selection rules implied by the operator $J_{y}^{m}$, not all intermediate states, expressed as a sum of $n$-particle and $v$-hole states, are allowed and thus the specification of the initial and final states determines the nature of the intermediate states.

5.1 Basic QUANTITIES. - 5.1.1 $X_{n K}$ and $X$. We have shown that the quantity $X$ is closely related to $X_{n K}$ which is the expectation value of the operator $J_{y}^{2}$ with respect to the intrinsic state $|n K\rangle$.

In the case $|n K\rangle$ represents the ground state vacuum $|0\rangle, X_{n K}$ is given by

$$
X_{n K}=2\left\langle n K\left|J_{y}^{2}\right| n K\right\rangle=2 \sum_{i \alpha}\left|\left\langle J_{y}\right\rangle_{i \alpha}\right|^{2},
$$

whereas, if $|n K\rangle$ represents the one-particle one-hole state, $a_{p}^{+} b_{\alpha}^{+}|0\rangle$, we have

$$
\begin{aligned}
X_{n K}=\sum_{k \gamma}^{\prime} \mid & \left.\left\langle J_{y}\right\rangle_{k \gamma}\right|^{2}+\left|\left\langle J_{y}\right\rangle_{p \alpha}\right|^{2}+ \\
& +\sum_{k}\left|\left\langle J_{y}\right\rangle_{p k}\right|^{2}+\sum_{\gamma}\left|\left\langle J_{y}\right\rangle_{\alpha \gamma}\right|^{2} .
\end{aligned}
$$

The prime on the sigma denotes the omission of the terms $k=p$ and $\gamma=\alpha$ from the summation.

A simple but important case is the one-particle states of an even-odd nucleus for $|n K\rangle$, which is constructed by applying a particle creation operator to the vacuum represented by the neighbouring eveneven nucleus. For $|n K\rangle=a_{p}^{+}|0\rangle$, we have

$$
\begin{aligned}
X_{n K}=2 \sum_{i \alpha}\left|\left\langle J_{y}\right\rangle_{i \alpha}\right|^{2}+ & 2 \sum_{i}\left|\left\langle J_{y}\right\rangle_{p i}\right|^{2}- \\
& -2 \sum_{\alpha}\left|\left\langle J_{y}\right\rangle_{p \alpha}\right|^{2} .
\end{aligned}
$$

The first term is nothing but the contribution from the core, which is the neighbouring even-even nucleus, and two other terms represent corrections arising from the removal of a one-particle state from the core. When we put $X_{n K}=X+\Delta X_{n K}$ with $\Delta X_{n K}=-a_{K K}^{(2)+}$, we may assume that the $X$ represents a core contribution and $\Delta X_{n K}$ is a small correction. In most cases, $\Delta X_{n K}$ is seen to be small and we have in particular $X=X_{n K}$ for the ground-state band of an eveneven nucleus.

5.1.2 $\left\langle H J_{y}^{2}\right\rangle_{i i}$ and $\left\langle H i J_{y}\right\rangle_{i-i^{*}}$ - The first order energy correction which contains two basic matrix elements $\left\langle H J_{y}^{2}\right\rangle$ and $\left\langle H i J_{y}\right\rangle$ is very instructive for understanding the meaning of various matrix elements in the different orders of the expansion. The first part of equation (25), for which we use the notation $E_{K}^{(1)+}$ in accordance with the meaning of the $\operatorname{sign}(+)$, is now

$$
\begin{aligned}
E_{\mathbf{K}}^{(1)+}=-2 \sum_{n^{\prime} \mathbf{K}^{\prime}}^{\prime}\langle n K|H| & \left.n^{\prime} K^{\prime}\right\rangle \times \\
& \times\left\langle n^{\prime} K^{\prime}\left|J_{\boldsymbol{y}}^{2}\right| n K\right\rangle,
\end{aligned}
$$

where the prime on the summation denotes that $\left(n^{\prime} K^{\prime}\right) \neq(n K)$. The above form of the expression appears throughout the present theory and we therefore treat it with several specific assumptions about the intrinsic state $|n K\rangle$. Because of the operators $H$ and $J_{y}^{2}$, the choice of the intermediate states follows from the nature of $|n K\rangle$. For example, the constant operator of $J_{y}^{2}$ in equation (46) as well as the kinetic energy part of equation (44) give no contributions to $E_{K}^{(1)+}$.

a) Even-even nuclei, - An interesting case is when $|n K\rangle$ represents the vacuum with $K=0$. The allowed intermediate states $\left|n^{\prime} K^{\prime}\right\rangle$ are then either oneparticle one-hole states or two-particle two-hole states. But, the one-particle one-hole states give no contribution, since the matrix elements of the Hamiltonian between the vacuum and one-particle onehole states vanish in the present approximation. As a consequence

$$
E_{K}^{(1)+}=-2 \sum_{i k \alpha \beta} V_{\alpha \beta, k i}\left\langle J_{y}\right\rangle_{i \beta}\left\langle J_{y}\right\rangle_{k \alpha}
$$

This interaction term is represented by figure 1 . The 


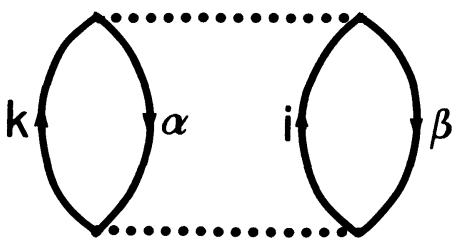

Fig. 1. - The diagram representation of $E_{\mathbf{K}}^{(1)+}$ for the ground state vacuum, $|n K\rangle=|0\rangle$.

intrinsic state $|n K\rangle$ may be, for example, an excited band such as one-particle one-hole state or twoparticle two-hole state. In the former case, we can also express $E_{\mathrm{K}}^{(1)+}$ in terms of $V$ and $J_{y}$ and this is perform- ed in Appendix $G$ where we have also shown in figure 6 the diagramatic representation for the oneparticle one-hole case.

b) Even-odd nuclei. - A simple form for the intrinsic state $|n K\rangle$ for an even-odd nucleus is the oneparticle state $a_{p}^{+}|0\rangle$, generated out of the neighbouring even-even nucleus core. The allowed intermediate states then consist of one-particle states, two-particle one hole states and three-particle two-hole states. Of these the one-particle states which should be different from the one-particle state of $|n K\rangle$ cannot contribute to $E_{K}^{(1)+}$ in the present approximation. The contributions from the two-particle one-hole states and from the three-particle two-hole states yield

$$
\begin{aligned}
E_{\mathrm{K}}^{(1)+}=-2 \sum_{i k \alpha \beta} V_{\alpha \beta, k i}\left\langle J_{y}\right\rangle_{k \alpha}\left\langle J_{y}\right\rangle_{i \beta} & -2\left\{\sum_{i \alpha} V_{p \alpha, p i}\left(\sum_{r}\left\langle J_{y}\right\rangle_{i r}\left\langle J_{y}\right\rangle_{r \alpha}-\sum_{\rho}\left\langle J_{y}\right\rangle_{i \rho}\left\langle J_{y}\right\rangle_{\rho \alpha}\right)+\right. \\
& \left.+2 \sum_{i j \alpha} V_{p \alpha, i j}\left\langle J_{y}\right\rangle_{i p}\left\langle J_{y}\right\rangle_{j \alpha}+2 \sum_{i \alpha \beta} V_{\alpha \beta, i p}\left\langle J_{y}\right\rangle_{p \alpha}\left\langle J_{y}\right\rangle_{i \beta}\right\} .
\end{aligned}
$$

These four terms are represented by figure 2 . The first term, illustrated in figure $2 a$ is nothing but the contribution from the core. As will be seen later, the inertia parameter of an even-even nucleus is directly related by the first term of equation (53) and it thus implies that the next three terms can be interpreted as correction to this parameter, arising from the change from the even-even nucleus to the even-odd nucleus. This point will be discussed again in the next section.

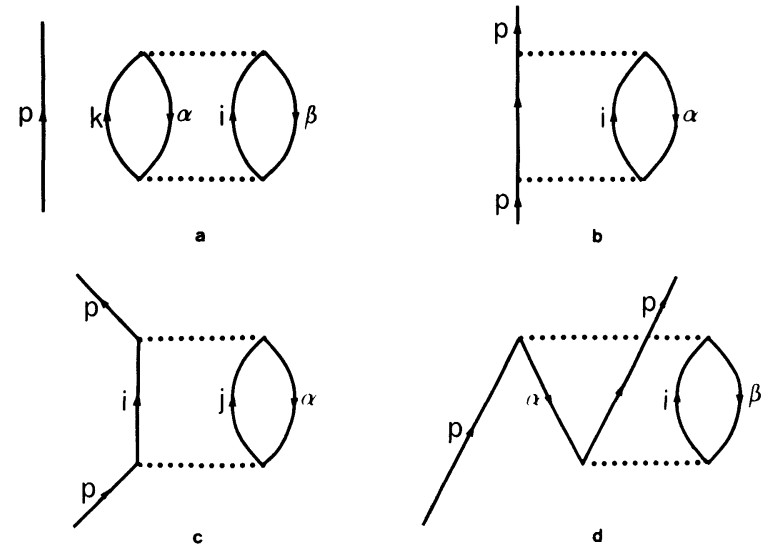

Fig. 2. - The diagram representation of $E_{K}^{(1)+}$ for the oneparticle state, $|n K\rangle=a_{p}^{+}|0\rangle$.

The second part of equation (25), which we shall denote $E_{K}^{(1)-}$, can be evaluated in the same manner as in derivation of equation (53), except that the intermediate three-particle two-hole states are not allowed because of the one-body operator character of $J_{y}$. We have thus

$$
E_{K}^{(1)-}=2 \delta_{K \frac{1}{2}}(-1)^{J+\frac{1}{2}}\left(J+\frac{1}{2}\right) \sum_{k \alpha} V_{p \alpha, \bar{p} k}\left\langle i J_{y}\right\rangle_{k x}
$$

The interaction matrix element $V_{p \alpha, p \bar{k}}$ is shown in figure 3. In deriving this result, we have used the timereversed state

$$
|n-K\rangle=\exp \left(-i \pi J_{y}\right)|n K\rangle=a_{\bar{p}}^{+}|0\rangle .
$$

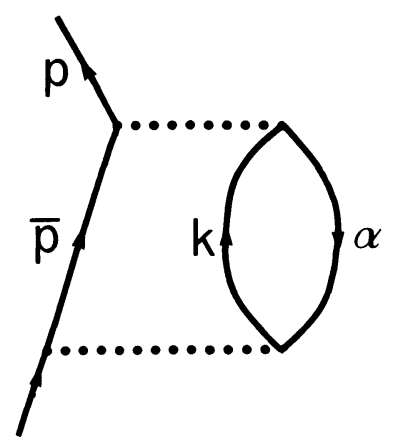

Fig. 3. - The diagram representation of $E_{\mathbf{K}}^{(1)-}$ for the oneparticle state, $|n K\rangle=a_{p}^{+}|0\rangle$.

The energy $E_{K}^{(1)-}$ is related to the decoupling parameter.

5.1.3 Decoupling parameter. - By analogy with the phenomenological energy formula in which the decoupling parameter, $a$, appears,

$$
E_{\mathbf{K}}^{J}=E_{\mathbf{K}}+A\left\{J(J+1)+a \delta_{K \frac{1}{2}}(-1)^{J+\frac{1}{2}}\left(J+\frac{1}{2}\right)\right\},
$$


with $a=-\left\langle\frac{1}{2}\left|J_{+}\right|-\frac{1}{2}\right\rangle$, we can express the corresponding decoupling parameter in the present formalism. This can be accomplished by combining the first order energy correction $E_{K}^{(1)}$ - of equation (54) and the term containing the factor $J(J+1)-K^{2}$ in the second order energy correction. Thus

$$
\begin{aligned}
a & =-X \frac{\left\langle H i J_{y}\right\rangle_{i-i}}{\left\langle H J_{y}^{2}\right\rangle_{i i}} \\
& =\frac{2 \sum_{i \alpha}\left|\left\langle J_{y}\right\rangle_{i \alpha}\right|^{2} \sum_{k \beta}\left\langle i J_{y}\right\rangle_{k \beta} V_{p \beta, \bar{p} k}}{\sum_{i k \alpha \beta}\left\langle J_{y}\right\rangle_{i \beta}\left\langle J_{y}\right\rangle_{k \alpha} V_{\alpha \beta, k i}} .
\end{aligned}
$$

This microscopic decoupling parameter is to be compared also with the calculation performed within the formalism of the approximate projection method [26]. When we assume that the two-body interaction part in the denominator has approximately equal magnitude to that of the numerator, equation (56) is roughly proportional to $-2\left\langle i J_{y}\right\rangle$, which is the phenomenological result for the decoupling parameter.

5.2 Moment of Inertia. - The second order energy correction has a term which is proportional to $J(J+1)-K^{2}$ and which may be simply written as

$$
-\left\{J(J+1)-K^{2}\right\}\left\{\frac{1}{X^{2}} \cdot E_{K}^{(1)+}\right\} .
$$

In accordance with the phenomenological model the factor multiplying $J(J+1)$ is generally interpreted as the moment of inertia factor. It has been shown [15] that the self-consistent moment of inertia of Thouless and Valatin [31] reduces under certain conditions to the moment of inertia of equation (57). There exist various ways of obtaining expressions similar to equation (57). For example, it has been shown that the Yoccoz formula of the moment of inertia follows also from the classical statistical mechanics [27].

For even-even nuclei, the moment of inertia parameter for the ground state band can be expressed as

$$
\begin{aligned}
\frac{\hbar^{2}}{2 \Theta_{0}}=\frac{1}{2} \frac{1}{\left(\sum_{k \alpha}\left|\left\langle J_{y}\right\rangle_{k \alpha}\right|^{2}\right)^{2}} \times \\
\quad \times \sum_{i k \alpha \beta} V_{\alpha \beta, k i}\left\langle J_{y}\right\rangle_{k \alpha}\left\langle J_{y}\right\rangle_{i \beta},
\end{aligned}
$$

where use has been made of the result (52). The modification to equation (58) has been proposed in a number of different ways [28, 31] in which additional assumptions have been made for the intrinsic states. The discussion on the relationship between equation (58) and the Inglis formula [29] is very useful.
$\mathrm{Hu}$ [30] and also Rouhaninejad and Yoccoz [12] have related the Inglis formula to that of PeierlsYoccoz.

Let us first note that the Hamiltonian $H$ commutes with $J_{y}$ in every basis. In the Hartree-Fock approximation, that is, when we use equations (44) and (45) in the commutator $\left[H, J_{y}\right]=0$, we obtain then three equations amongst which one is particularly interesting at this stage. It takes the form

$$
\begin{aligned}
& \left(\varepsilon_{k}-\varepsilon_{\alpha}\right)\left\langle J_{y}\right\rangle_{\alpha k}+ \\
& \quad+\sum_{i \beta}\left\{\left\langle J_{y}\right\rangle_{\beta i} V_{\alpha i, k \beta}-\left\langle J_{y}\right\rangle_{i \beta} V_{\alpha \beta, k i}\right\}=0 .
\end{aligned}
$$

Thouless [31] has already considered this kind of equation in different forms. The first interaction term with $V$ corresponds to the one-particle one-hole interaction and the second interaction term corresponds to the two-particle two-hole interaction. When we relate equation (59) to equation (58), we get

$$
\begin{aligned}
& \frac{\hbar^{2}}{2 \Theta_{0}}=\frac{2}{X^{2}} \sum_{k \alpha}\left\langle J_{y}\right\rangle_{k \alpha} \times \\
& \quad \times\left\{\left(\varepsilon_{k}-\varepsilon_{\alpha}\right)\left\langle J_{y}\right\rangle_{\alpha k}+\sum_{i \beta}\left\langle J_{y}\right\rangle_{\beta i} V_{x i, k \beta}\right\} .
\end{aligned}
$$

When we neglect the one-particle one-hole interaction, equation (60) reduces, in unit of $\hbar^{2}$, to

$$
\Theta_{0}=\frac{\left(\sum_{k \alpha}\left|\left\langle J_{y}\right\rangle_{k x}\right|^{2}\right)^{2}}{\sum_{k \alpha}\left(\varepsilon_{k}-\varepsilon_{x}\right)\left|\left\langle J_{y}\right\rangle_{k \alpha}\right|^{2}} .
$$

This result is to be compared with that of Inglis. There is however, a priori, no reason that the second term in equation (60) is neglected, except that the kinetic energy part is much larger than the one-particle onehole interaction. An alternative way of approximating the interaction $V_{\alpha \beta, k i}$ in equation (58) was shown in reference [12].

In a similar way, we can also express the inertia parameter of even-odd nuclei in terms of the twobody interaction and this can be accomplished using the results (53) and (57). However, what is interesting is the amount of change brought about by the transition from the even-even nucleus to a neighbouring even-odd nucleus which is now supposed to be a one-particle state generated from the core. Since $E_{\mathbf{K}}^{(1)+}$ for the even-even nucleus is composed of the contribution from the core and the correction due to the addition of an extra particle, the difference between the inertia parameter of the even-odd nucleus and that of the even-even nucleus is given by

$$
\begin{aligned}
& R \frac{\hbar^{2}}{2 \Theta}-\frac{\hbar^{2}}{2 \Theta_{0}}=\frac{1}{2} \frac{1}{\left\{\sum_{k \alpha}\left|\left\langle J_{y}\right\rangle_{k \alpha}\right|^{2}\right\}^{2}}\left\{\sum_{l \alpha} V_{p \alpha, p i}\left(\sum_{r}\left\langle J_{y}\right\rangle_{i r}\left\langle J_{y}\right\rangle_{r \alpha}-\sum_{\rho}\left\langle J_{y}\right\rangle_{i \rho}\left\langle J_{y}\right\rangle_{\rho \alpha}\right)+\right. \\
& \left.+2 \sum_{i k \alpha} V_{p \alpha, i k}\left\langle J_{y}\right\rangle_{i p}\left\langle J_{y}\right\rangle_{k \alpha}+2 \sum_{i \alpha \beta} V_{\alpha \beta, i p}\left\langle J_{y}\right\rangle_{p \alpha}\left\langle J_{y}\right\rangle_{i \beta}\right\}
\end{aligned}
$$


where

$$
R=\left(1+\frac{\Delta X}{X(\text { core })}\right)^{2} \quad \text { with } \quad \Delta X=X-X(\text { core })
$$

When we assume that $\Delta X=X_{n K}-X_{n K}$ (core), we see that $\Delta X$ amounts to $2\left\{\sum_{i}\left|\left\langle J_{y}\right\rangle_{p i}\right|^{2}-\sum_{x}\left|\left\langle J_{y}\right\rangle_{p \alpha}\right|^{2}\right\}$ which can be really small. For many cases, the ratio $\Delta X / X$ (core) may therefore be small and thus the assumption of $R \simeq 1$ is very plausible. From the phenomenological point of view, the variation of moment of inertia, due to the addition or subtraction of a nucleon from the core, cannot be estimated uniquely and it is necessary to make additional assumptions to those of the rotational model. In contrast, equation (62) allows us to calculate the change of the moment of inertia without assuming other supplementary conditions.

5.3 BAND-MIXING. $-5.3 .1\left|K_{i}-K_{l}\right|=1$ or $K_{i}+K_{l}=1$. - Let us first consider the band-mixing between the ground state band $K_{i}=0$ and the excited band $K_{l}=1$ which is supposed to be one-particle onehole state. The band mixing term is given by

$$
\begin{aligned}
\delta_{K_{l} 1} \frac{J(J+1)}{X^{2}\left(E_{K_{i}}^{(0)}-E_{K_{l}}^{(0)}\right)} \mid \sum_{k \beta}\left\{\left(\left\langle i J_{y}\right\rangle_{k \beta} V_{\alpha \beta, k p}\right.\right. & \left.+\left\langle i J_{y}\right\rangle_{\beta k} V_{k \alpha, p \beta}\right)+(-1)^{J+1} \times \\
& \times(\text { the same terms but with } \bar{\alpha} \text { and } \bar{p} \text { instead of } \alpha \text { and } p)\}\left.\right|^{2},
\end{aligned}
$$

where we have assumed that $\left|n_{i} K_{i}\right\rangle=|0\rangle,\left|n_{l} K_{l}\right\rangle=a_{p}^{+} b_{\alpha}^{+}|0\rangle$ and $\left|n_{l}-K_{l}\right\rangle=a_{\bar{p}}^{+} b_{\bar{x}}^{+}|0\rangle$. In the above expression, the terms with the phase $(-1)^{J+1}$ are for $K_{i}+K_{l}=1$. It is not difficult to obtain similar expressions using $\left|n_{l} K_{l}\right\rangle=a_{p}^{+} a_{q}^{+} b_{\alpha}^{+} b_{\beta}^{+}|0\rangle$. The first part of interactions in the expressions (63) are illustrated in figure 4. The intrinsic state $\left|n_{i} K_{i}\right\rangle$ may also be one-particle one-hole or two-particle two-hole states of the excited bands. In Appendix $H$ we show the one-particle one-hole case for both $\left|n_{i} K_{i}\right\rangle$ and $\left|n_{l} K_{l}\right\rangle$.

In equation (39), we have formally seen how the band-mixing effect has influence on the moment of inertia. The quantitative calculation of this effect can now be performed by substituting the result (63) into equation (39.3). The modified moment of inertia may be increased or decreased with respect to the initial value, depending on whether the sign of the denominator $E_{K_{i}}^{(0)}-E_{K_{l}}^{(0)}$ is positive or negative. The numerical result will be given in a forthcoming paper in relation to specific nuclei.

For even odd nuclei, the simplest case is the one-particle state for both $\left|n_{i} K_{i}\right\rangle$ and $\left|n_{l} K_{l}\right\rangle$, such as $\left|n_{i} K_{i}\right\rangle=a_{p}^{+}|0\rangle$ and $\left|n_{l} K_{l}\right\rangle=a_{q}^{+}|0\rangle$. The band-mixing term is then

$$
\begin{aligned}
\frac{1}{X^{2}\left(E_{K_{i}}^{(0)}-E_{K_{l}}^{(0)}\right)} \mid \sum_{k \alpha}\left[ \pm \delta_{K_{i}, K_{l} \pm 1}\right. & \sqrt{J(J+1)-K_{i} K_{l}}\left\{\left\langle i J_{y}\right\rangle_{\alpha k} V_{p k, q \alpha}+\left\langle i J_{y}\right\rangle_{k \alpha} V_{p \alpha, q k}\right\}- \\
& \left.-\delta_{K_{i} \frac{1}{2}} \delta_{K_{l} \frac{1}{2}}(-1)^{J+\frac{1}{2}}\left(J+\frac{1}{2}\right)\left\{\left\langle i J_{y}\right\rangle_{\alpha k} V_{p k, \bar{q} \alpha}+\left\langle i J_{y}\right\rangle_{k \alpha} V_{p \alpha, \bar{q} k}\right\}\right]\left.\right|^{2}
\end{aligned}
$$

where time-reversed states are those for which $K_{i}+K_{l}=1$. The first part of the interactions in equation (64) is illustrated in figure 5. One essential difference between the formula (64) and the phenomenological band-mixing term with a Coriolis force [32] is that the moment of inertia does not appear as a constant multiplier in the microscopic expression but is distributed over the expression (64) and so is able to take into account the change of the
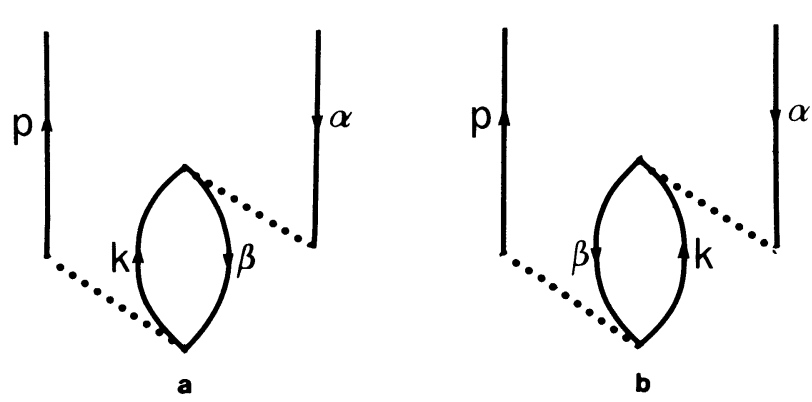

Fig. 4. - The diagram representation of the band-mixing matrix element between the ground state vacuum, $\left|n_{i} K_{i}\right\rangle=|0\rangle$, and the excited one-particle one-hole state, $\left|n_{l} K_{l}\right\rangle=a_{p}^{+} b_{\alpha}^{+}|0\rangle$.
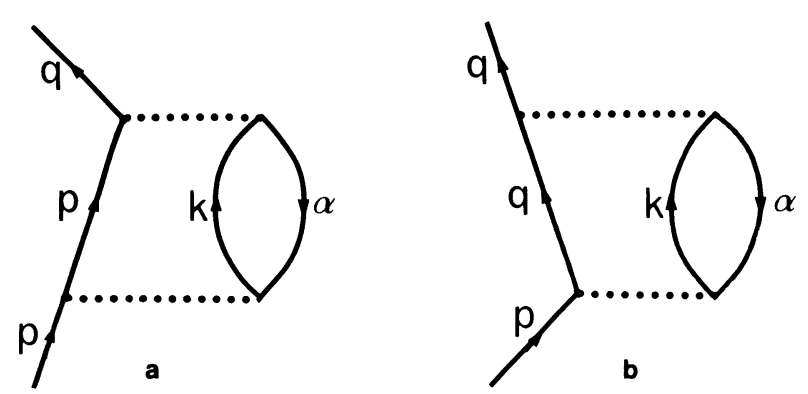

Fig. 5. - The diagram representation of the band-mixing matrix element for $|\Delta K|=1$ between the one-particle states, $\left|n_{i} K_{i}\right\rangle=a_{p}^{+}|0\rangle$ and $\left|n_{l} K_{l}\right\rangle=a_{q}^{+}|0\rangle$. 
moment of inertia arising from band-mixing. When we assume that equation (57) is equally valid for the evenodd nucleus, the band-mixing term without the factor $J(J+1)-K_{i} K_{l}$ becomes, in the absence of $K=\frac{1}{2}$ bands,

$$
\frac{X^{2}}{4} \frac{\left|\sum_{k \alpha}\left\{\left\langle i J_{y}\right\rangle_{\alpha k} V_{p k, q \alpha}+\left\langle i J_{y}\right\rangle_{k \alpha} V_{p \alpha, q k}\right\}\right|^{2}}{\left|\sum_{i k \alpha \beta}\left\langle J_{y}\right\rangle_{i \beta}\left\langle J_{y}\right\rangle_{k \alpha} V_{\alpha \beta, k i}\right|^{2}} .
$$

This is the term which is to be compared with the phenomenological coupling term $\left|\left\langle K_{l}\left|J_{+}\right| K_{i}\right\rangle\right|^{2}$. Since the quantity $X$ is about equal to $2 \sum\left|\left\langle J_{y}\right\rangle\right|^{2}$, the expression (65) is approximately equal to $\left|2\left\langle J_{y}\right\rangle\right|^{2}$ which has the same order of magnitude as the strong coupling formula.

5.3.2 $\left|K_{i}-K_{l}\right|=2$ or $K_{i}+K_{l}=2$. - Two simple cases are the mixing between the ground band and the excited band with $K=2$. The $K=2$ band may be represented by one-particle one-hole or two-particle two-hole states for even-even nuclei and one-particle states for the simple case of even-odd nuclei.

For $\left|n_{i} K_{i}\right\rangle=|0\rangle$ and $\left|n_{l} K_{l}=2\right\rangle=a_{p}^{+} b_{a}^{+}|0\rangle$ of even-even nuclei, we have from equation (36)

$$
\begin{aligned}
M_{i}^{(4)}=\sqrt{J(J+1)[J(J+1)-2]}[ & \left\{\sum_{i \beta} V_{\alpha \beta, p i}\left(\sum_{r}\left\langle J_{y}\right\rangle_{i r}\left\langle J_{y}\right\rangle_{r \beta}-\sum\left\langle J_{y}\right\rangle_{i \sigma}\left\langle J_{y}\right\rangle_{\sigma \beta}\right)+\right. \\
& +2 \sum_{i k \beta} V_{\alpha \beta, i k}\left\langle J_{y}\right\rangle_{i p}\left\langle J_{y}\right\rangle_{k \beta}-2 \sum_{i \beta \gamma} V_{\beta \gamma, i p}\left\langle J_{y}\right\rangle_{i \beta}\left\langle J_{y}\right\rangle_{\alpha \gamma} \\
& +\sum_{i \beta} V_{i \alpha, \beta p}\left(\sum_{r}\left\langle J_{y}\right\rangle_{\beta r}\left\langle J_{y}\right\rangle_{r i}-\sum_{\sigma}\left\langle J_{y}\right\rangle_{\beta \sigma}\left\langle J_{y}\right\rangle_{\sigma i}\right) \\
& \left.+2 \sum_{i k \beta} V_{i k, p \beta}\left\langle J_{y}\right\rangle_{\alpha i}\left\langle J_{y}\right\rangle_{\beta k}-2 \sum_{i \beta \gamma} V_{i \alpha, \beta \gamma}\left\langle J_{y}\right\rangle_{\beta i}\left\langle J_{y}\right\rangle_{\gamma p}\right\} \\
& +\{\text { the same terms but with } \bar{p} \text { and } \bar{\alpha} \text { in place of } p \text { and } \alpha\}],
\end{aligned}
$$

where the time-reversed states are for the case with $K_{i}+K_{l}=2$.

For $\left|n_{i} K_{i}\right\rangle=a_{p}^{+}|0\rangle$ and $\left|n_{l} K_{l}\right\rangle=a_{q}^{+}|0\rangle$ for even-odd nuclei we have instead

$$
\begin{aligned}
& \frac{1}{X^{4}} \mid G_{1}\left\{\sum_{k \alpha} V_{p \alpha, q k}\left(\sum_{r}\left\langle J_{y}\right\rangle_{k r}\left\langle J_{y}\right\rangle_{r \alpha}-\sum_{\beta}\left\langle J_{y}\right\rangle_{k \beta}\left\langle J_{y}\right\rangle_{\beta \alpha}\right)+\right. \\
&+2 \sum_{i k \alpha} V_{p \alpha, i k}\left\langle J_{y}\right\rangle_{i q}\left\langle J_{y}\right\rangle_{k \alpha}+2 \sum_{k \alpha \beta} V_{\beta \alpha, k q}\left\langle J_{y}\right\rangle_{p \beta}\left\langle J_{y}\right\rangle_{k \alpha} \\
&+ \sum_{k \alpha} V_{p k, q \alpha}\left(\sum_{r}\left\langle J_{y}\right\rangle_{\alpha r}\left\langle J_{y}\right\rangle_{r k}-\sum_{\beta}\left\langle J_{y}\right\rangle_{\alpha \beta}\left\langle J_{y}\right\rangle_{\beta k}\right) \\
&\left.+2 \sum_{i k \beta} V_{i k, q \alpha}\left\langle J_{y}\right\rangle_{p i}\left\langle J_{y}\right\rangle_{\alpha k}+2 \sum_{k \alpha \beta} V_{p k, \alpha \beta}\left\langle J_{y}\right\rangle_{\alpha k}\left\langle J_{y}\right\rangle_{\beta q}\right\} \\
&+\left.G_{2}\{\text { terms with } \bar{q} \text { in place of } q\}\right|^{2},
\end{aligned}
$$

with

$$
\begin{aligned}
& G_{1}=\delta_{K_{1}, K_{i} \pm 2} \sqrt{\left[J(J+1)-K_{i}\left(K_{i} \pm 1\right)\right]\left[J(J+1)-\left(K_{i} \pm 1\right)\left(K_{i} \pm 2\right)\right]}, \\
& G_{2}=\delta_{K_{i}+K_{l}, 2}\left[\delta_{K_{i}\left\{\begin{array}{l}
0 \\
2
\end{array}\right\}}(-1)^{J} \sqrt{J(J-1)(J+1)(J+2)} \pm\right. \\
& \left. \pm \delta_{K_{i}\left\{\begin{array}{l}
1 / 2\} \\
3 / 2
\end{array}\right\}}(-1)^{J+\frac{1}{2}}\left(J+\frac{1}{2}\right) \sqrt{\left(J+\frac{3}{2}\right)\left(J-\frac{1}{2}\right)}+\delta_{K_{i} 1}(-1)^{J+1} J(J+1)\right] .
\end{aligned}
$$

Actually, the expression (67) has to be divided by the energy difference between the two bands, $E_{K_{i}}^{(0)}-E_{K_{l}}^{(0)}$. The first and third factors of $G_{2}$ are valid only for even-even nuclei.

5.4 SPECIFIC TERMS. - As shown in equations (36) and (38), in third and fourth order energy corrections, we have terms having the factors $\delta_{K \frac{3}{2}}(-1)^{J+3 / 2}\left(J+\frac{3}{2}\right)\left(J+\frac{1}{2}\right)\left(J-\frac{1}{2}\right)$ and $\delta_{K_{\frac{1}{2}}}(-1)^{J+1 / 2}\left(J+\frac{1}{2}\right) J(J+1)$, 
respectively. The specific term to the $K=\frac{3}{2}$ band contains the operator $H i J_{y}^{3}$, while the terms having the latter factor contain in addition operators like $\mathrm{HJ}_{y}^{2}$ and $\mathrm{HiJ}_{\mathrm{y}}$ and thus the expression of these terms as functions of two-body interaction are rather lengthy. In Appendix I, we explicitly present the matrix element $\left\langle\mathrm{HiJ}_{y}^{3}\right\rangle_{i-i}$ as an example of evaluation of the matrix element of the operator $J_{y}^{3}$. The fourth order energy correction has a term which is specific to $K=2$ bands and which has the matrix element of the operator $H J_{y}^{4}$. Such operators appear also in the term containing the factor $\left[J(J+1)-K^{2}\right]^{2}$. Because of the fourth power of the operator $J_{y}$, the calculation of the matrix element of the operator $H J_{v}^{4}$ is very cumbersome, except for a very few special cases. Thus for the ground state band of even-even nuclei, the term having the square of $J(J+1)$ is given by

$$
\begin{aligned}
& {[J(J+1)]^{2} \frac{2}{3 X^{4}} \sum_{i k \alpha \beta} V_{\alpha \beta, i k}\left\{4 \langle J _ { y } \rangle _ { k \beta } \left(\sum_{l m}\left\langle J_{y}\right\rangle_{i l}\left\langle J_{y}\right\rangle_{l m}\left\langle J_{y}\right\rangle_{m \alpha}\right.\right.} \\
& \left.+\sum_{\gamma \eta}\left\langle J_{y}\right\rangle_{i \gamma}\left\langle J_{y}\right\rangle_{\gamma \eta}\left\langle J_{y}\right\rangle_{\eta \alpha}\right)-8\left\langle J_{y}\right\rangle_{k \beta} \sum_{l \gamma}\left(\left\langle J_{y}\right\rangle_{i l}\left\langle J_{y}\right\rangle_{l y}\left\langle J_{y}\right\rangle_{\gamma \alpha}\right. \\
& \left.+\left\langle J_{y}\right\rangle_{i \gamma}\left\langle J_{y}\right\rangle_{\gamma l}\left\langle J_{y}\right\rangle_{l \alpha}\right)+3\left(\sum_{l}\left\langle J_{y}\right\rangle_{k l}\left\langle J_{y}\right\rangle_{l \beta}-\sum_{\gamma}\left\langle J_{y}\right\rangle_{k \gamma}\left\langle J_{y}\right\rangle_{\gamma \beta}\right) \\
& \left.\times\left(\sum_{m}\left\langle J_{y}\right\rangle_{i m}\left\langle J_{y}\right\rangle_{m \alpha}-\sum_{\eta}\left\langle J_{y}\right\rangle_{i \eta}\left\langle J_{y}\right\rangle_{\eta \alpha}\right)\right\} \text {. }
\end{aligned}
$$

The interaction factor $V_{\alpha \beta, i k}$ has already been illustrated in figure 1. It has been shown [27] that the use of the classical partition function leads to the ensemble average $\left\langle J^{4}\right\rangle=2\left\langle J^{2}\right\rangle^{2}$. This relation may be applied to the estimation of the expectation value $\left\langle J_{y}^{4}\right\rangle_{i i}$. For even-odd nuclei we can also obtain a similar expression for the term having the factor $\left[J(J+1)-K^{2}\right]^{2}$.

6. Summary and conclusion. - A microscopic description of rotational spectra with band-coupling we have presented here is an attempt of exposing the rotational ingredients contained in the projection method and therefore, in a sense, an extension of the Peierls-Yoccoz theory of collective motion.

Most approaches to handle the complicated variational equations of projected state, which cannot be treated exactly, yielded $[14,15]$ the cranking model equations as a first approximation. We have, however, not attempted to study the rotational projection by solving the Hartree-Fock equation in the cranking model. Instead, we have first extracted various rotational constituents with axial symmetry from the projected energy formula. This has been accomplished by expanding both the overlap and energy integrals in the projected energy expression in inverse powers of the quantity $X$, which is large and is something like an averaged quantity of the expectation value of $J_{y}^{2}$ over several bands in consideration. We have next remarked that, when the same expansion is also allowed for the variational equations of the projected state, then the lowest order equation reduces to the ordinary Hartree-Fock equation and that the first order equation can be solved provided that the zero order solution is known. This argument shows clearly to what degree the approach, in which the ordinary Hartree-Fock solutions are employed in the projected rotational energy formula, is reliable. While the consideration of higher order solutions would not be necessary owing to the smallness of $1 / X$ in powers, it is to be desired that the individual wave functions are at least those solutions from the first order equation. The assumption of large deformation is therefore a fundamental requirement so as to have confidence in the present formulation. The particle-hole formalism in a selfconsistent basis that we have introduced in the last part is to show a method of microscopic calculations of various rotational quantities derived in the first part. It is to be noticed that the projected energy of equation (19) as a whole can be minimized with respect to individual functions when the model space has a manageable dimension.

Much of the present results are compared with the corresponding terms in the phenomenological rotational model. Besides the well known terms such as the moment of inertia parameter, the decoupling factor and the band-mixing matrix element for $|\Delta K|=1$, we have obtained other unfamiliar quantities having the factors with peculiar phases,

$$
\begin{gathered}
(-1)^{J+1} J(J+1),(-1)^{J+\frac{3}{2}\left(J-\frac{1}{2}\right)\left(J+\frac{1}{2}\right)\left(J+\frac{3}{2}\right),} \\
(-1)^{J} J(J+1)(J-1)(J+2)
\end{gathered}
$$

and

$$
\left[J(J+1)-K^{2}\right]^{2}
$$

The band-mixing matrix element for $|\Delta K|=2$ is also a new formula.

The perturbation expansion with which we have established the band-mixing matrix elements would not affect the reliability of the present approach, except for the case where the rotational bands in 
question are very close to each other. Inclusion of the pairing field may in principle be possible with some changes but this is beyond the scope of the present study.

Numerical calculations for some specific nuclei will be given in a forthcoming paper.
Acknowledgments. - The authors would like to thank Professor J. Yoccoz who introduced us several techniques used in the present calculation. We would also like to thank the staff of the theoretical physics group of the Nuclear Science Institute of Grenoble for many helpful discussions.

Appendix $\mathbf{A}: a_{K K^{\prime}}^{(m) \pm}$ and $\varepsilon_{K K^{\prime}}^{(m) \pm}$ - We first note the relation

$$
a_{K K^{\prime}}^{(m-2) \pm}=\frac{\partial}{\partial X} a_{K K^{\prime}}^{(m) \pm} \quad \text { for } \quad m \geqslant 2,
$$

which holds also for $\varepsilon_{K K^{\prime}}^{(m) \pm}$. The coefficients $a_{K K^{\prime}}^{(m) \pm}$ with $m=7$ and 8 are

$$
\begin{aligned}
a_{K K^{\prime}}^{(7) \pm}=\mp \frac{X^{3}}{3}\left\langle n K\left|i J_{y}\right| n^{\prime} \pm K^{\prime}\right\rangle \pm \frac{2}{3} X^{2}\left\langle n K\left|i J_{y}^{3}\right| n^{\prime} \pm K^{\prime}\right\rangle \mp & \\
& \mp \frac{4}{15} X\left\langle n K\left|i J_{y}^{5}\right| n^{\prime} \pm K^{\prime}\right\rangle \pm \frac{8}{315}\left\langle n K\left|i J_{y}^{7}\right| n^{\prime} \pm K^{\prime}\right\rangle, \\
a_{K K^{\prime}}^{(8) \pm}=\frac{1}{24} X^{4} a_{K K^{\prime}}^{(0) \pm}-\frac{X^{3}}{3}\left\langle n K\left|J_{y}^{2}\right| n^{\prime} \pm K^{\prime}\right\rangle+ & \frac{X^{2}}{3}\left\langle n K\left|J_{y}^{4}\right| n^{\prime} \pm K^{\prime}\right\rangle- \\
& -\frac{4}{45} X\left\langle n K\left|J_{y}^{6}\right| n^{\prime} \pm K^{\prime}\right\rangle+\frac{2}{315}\left\langle n K\left|J_{y}^{8}\right| n^{\prime} \pm K^{\prime}\right\rangle .
\end{aligned}
$$

The energy coefficients $\varepsilon_{K K^{\prime}}^{(m) \pm}$ can be obtained from $a_{K K^{\prime}}^{(m) \pm}$ by replacing $J_{y}^{m}$ in the above formulas by $H J_{y}^{m}$.

Appendix B : $\gamma_{K K^{\prime}}^{(2 m)}$ and $\alpha_{K K^{\prime}}^{(m) \pm}$. The coefficients $\gamma_{K K^{\prime}}^{(2 m)}$ with $m=2$ and 3 in the expansion of the Wigner rotation matrix $d_{K^{\prime}}^{J}(\beta)$ in equation (11) are

$$
\begin{aligned}
\gamma_{\mathrm{KK}^{\prime}}^{(4)}=\frac{1}{2\left(K-K^{\prime}\right.}+ & 1)\left(K-K^{\prime}+2\right)\left\{\left[J(J+1)-K K^{\prime}-\frac{1}{3}\left(K-K^{\prime}\right)\left(K-K^{\prime}+1\right)\right]^{2}-\right. \\
& -\frac{2}{3}\left[J(J+1)-K K^{\prime}-\frac{1}{3}\left(K-K^{\prime}\right)\left(K-K^{\prime}+1\right)\right]+K K^{\prime}\left(K-K^{\prime}+1\right) \\
& \left.+\frac{4}{45}\left(K-K^{\prime}\right)\left(K-K^{\prime}+1\right)\left[3\left(K-K^{\prime}\right)+1\right]\right\}, \\
\gamma_{\mathbf{K K}^{\prime}}^{(6)}=\frac{-1}{6\left(K-K^{\prime}\right.}+ & 1)\left(K-K^{\prime}+2\right)\left(K-K^{\prime}+3\right)\left\{\left[J(J+1)-K K^{\prime}-\frac{1}{3}\left(K-K^{\prime}\right)\left(K-K^{\prime}+1\right)\right]^{3}-\right. \\
& -2\left[J(J+1)-K K^{\prime}-\frac{1}{3}\left(K-K^{\prime}\right)\left(K-K^{\prime}+1\right)\right]^{2} \\
& +\frac{1}{5}\left[J(J+1)-K K^{\prime}-\frac{1}{3}\left(K-K^{\prime}\right)\left(K-K^{\prime}+1\right)\right]\left[4\left(K-K^{\prime}+2\right)\left(\left(K-K^{\prime}\right)^{2}+1\right)\right. \\
& \left.+25 K K^{\prime}+15 K K^{\prime}\left(K-K^{\prime}\right)\right]-\frac{4}{3} K K^{\prime}\left(K-K^{\prime}+1\right)\left(K-K^{\prime}+3\right) \\
& \left.-\frac{16}{189}\left(K-K^{\prime}\right)\left(K-K^{\prime}+1\right)\left(K-K^{\prime}+3\right)\left(4\left(K-K^{\prime}\right)+1\right)\right\} .
\end{aligned}
$$

The coefficients $\alpha_{K K^{\prime}}^{(m) \pm}$ can be deduced from equations (11) and (12) by putting $x^{K-K^{\prime}+2 m^{\prime}}=x^{m}$ for the case $K \geqslant K^{\prime} \geqslant 0$ and $x^{K+K^{\prime}+2 m^{\prime}}=x^{m}$ for the case $\beta^{\prime}=\pi-\beta$, and then evaluating the coefficients of $x^{m}$. We have

$$
\begin{aligned}
& \alpha_{K K}^{(0)+}=1 \\
& \alpha_{K K}^{(2)+}=-\left[J(J+1)-K^{2}\right], \\
& \alpha_{K K}^{(4)+}=\frac{1}{4}\left\{\left[J(J+1)-K^{2}\right]^{2}-\frac{2}{3}\left[J(J+1)-K^{2}\right]+K^{2}\right\}, \\
& \alpha_{K K}^{(6)+}=-\frac{1}{36}\left\{\left[J(J+1)-K^{2}\right]^{3}-2\left[J(J+1)-K^{2}\right]^{2}+\left(\frac{8}{5}+5 K^{2}\right)\left[J(J+1)-K^{2}\right]-4 K^{2}\right\},
\end{aligned}
$$




$$
\begin{aligned}
\alpha_{K K}^{(2 m)-} \delta_{K 0} & =(-1)^{J} \delta_{K 0} \alpha_{K K}^{(2 m)+}, \\
\alpha_{K, K+1}^{(1)+} & =\sqrt{J(J+1)-K(K+1)} \\
\alpha_{K, K+1}^{(3)+}=-\frac{1}{2} \sqrt{J(J+1)-K(K+1)}\left\{[J(J+1)-K(K+1)]-\frac{2}{3}\right\}, & \\
\alpha_{K, K+1}^{(5)+}=\frac{1}{12} \sqrt{J(J+1)-K(K+1)}\left\{[J(J+1)-K(K+1)]^{2}-2[J(J+1)-K(K+1)]+\right. & \left.+2 K(K+1)+\frac{8}{5}\right\},
\end{aligned}
$$

$\alpha_{K K^{\prime}}^{(2 m+1)-} \delta_{K+K^{\prime}, 1}=-(-1)^{J+K} \alpha_{K, K+1}^{(2 m+1)+}(-K$ in place of $K)$,

$$
\begin{aligned}
& \alpha_{K, K+2}^{(2)+}=\frac{1}{2} \sqrt{[J(J+1)-K(K+1)][J(J+1)-(K+1)(K+2)]}, \\
& \alpha_{K, K+2}^{(4)+}=-\frac{1}{6} \sqrt{[J(J+1)-K(K+1)][J(J+1)-(K+1)(K+2)]}\{[J(J+1)-K(K+2)]-2\},
\end{aligned}
$$

$\alpha_{K K^{\prime}}^{(2 m)-} \delta_{K+K^{\prime}, 2}=(-1)^{J+K} \alpha_{K, K+2}^{(2 m)+}(-K$ in place of $K)$.

Appendix C : Matrices $H^{(m)}$. - The matrices $H^{(2)}$ and $H^{(3)}$ in equation (21) are

$$
\begin{aligned}
& H^{(2)}=n^{(0)}\left\{h^{(2)}-\frac{1}{2} n^{(0)}\left[n^{(1)} h^{(1)}+h^{(1)} n^{(1)}\right]-\frac{1}{2} n^{(0)}\left[n^{(2)} h^{(0)}+h^{(0)} n^{(2)}\right]+\frac{1}{4}\left[n^{(0)}\right]^{2}\left[n^{(1)} h^{(0)} n^{(1)}\right]\right. \\
& \left.+\frac{3}{8}\left[n^{(0)}\right]^{2}\left[n^{(1)} n^{(1)} h^{(0)}+h^{(0)} n^{(1)} n^{(1)}\right]\right\}, \\
& H^{(3)}=n^{(0)}\left\{h^{(3)}-\frac{1}{2} n^{(0)}\left[n^{(1)} h^{(2)}+h^{(2)} n^{(1)}\right]-\frac{1}{2} n^{(0)}\left[n^{(2)} h^{(1)}+h^{(1)} n^{(2)}\right]\right. \\
& +\frac{1}{4}\left[n^{(0)}\right]^{2}\left[n^{(1)} h^{(1)} n^{(1)}\right]+\frac{3}{8}\left[n^{(0)}\right]^{2}\left[n^{(1)} n^{(1)} h^{(1)}+h^{(1)} n^{(1)} n^{(1)}\right] \\
& -\frac{1}{2} n^{(0)}\left[n^{(3)} h^{(0)}+h^{(0)} n^{(3)}\right]+\frac{1}{4}\left[n^{(0)}\right]^{2}\left[n^{(1)} h^{(0)} n^{(2)}+n^{(2)} h^{(0)} n^{(1)}\right] \\
& +\frac{3}{8}\left[n^{(0)}\right]^{2}\left[n^{(1)} n^{(2)} h^{(0)}+n^{(2)} n^{(1)} h^{(0)}+h^{(0)} n^{(1)} n^{(2)}+h^{(0)} n^{(2)} n^{(1)}\right] \\
& -\frac{1}{16}\left[n^{(0)}\right]^{3}\left[5\left[n^{(1)}\right]^{3} h^{(0)}+5 h^{(0)}\left[n^{(1)}\right]^{3}\right. \\
& \left.\left.+3 n^{(1)} h^{(0)}\left[n^{(1)}\right]^{2}+3\left[n^{(1)}\right]^{2} h^{(0)} n^{(1)}\right]\right\} \text {. }
\end{aligned}
$$

The matrix $H^{(4)}$ is rather lengthy and we do not present it here.

Appendix D: Functions $W$ and $L$. Some of functions $W$ and $L$ in equation (37) are

$$
\begin{aligned}
& W_{0}^{(2)}=\frac{4}{3}\left\langle H J_{y}^{4}\right\rangle_{i i}-2\left(X+2\left\langle J_{y}^{2}\right\rangle_{i i}-\frac{2}{3}\right)\left\langle H J_{y}^{2}\right\rangle_{i i}, \\
& W_{0}^{(3)}=-\frac{8}{15}\left\langle H J_{y}^{6}\right\rangle_{i i}+\frac{8}{3}\left(X+\left\langle J_{y}^{2}\right\rangle_{i i}-\frac{2}{3}\right)\left\langle H J_{y}^{4}\right\rangle_{i i}-2\left\{X^{2}+4 X\left\langle J_{y}^{2}\right\rangle_{i i}-\frac{4}{3}\left\langle J_{y}^{4}\right\rangle_{i i}+\right. \\
& \left.+4\left\langle J_{y}^{2}\right\rangle_{i i}^{2}-\frac{4}{3} X-\frac{8}{3}\left\langle J_{y}^{2}\right\rangle_{i i}+\frac{4}{45}+K_{i}^{2}\right\}\left\langle H J_{y}^{2}\right\rangle_{i i}, \\
& W_{J}^{(3)}=-\frac{8}{3}\left\langle H J_{y}^{4}\right\rangle_{i i}+\left(4 X+8\left\langle J_{y}^{2}\right\rangle_{i i}-\frac{4}{3}\right)\left\langle H J_{y}^{2}\right\rangle_{i i}, \\
& W_{1 / 2}^{(2)}=-4\left\langle i J_{y}\right\rangle_{i-i}\left\{\left\langle H J_{y}^{2}\right\rangle_{i i}+(-1)^{J+\frac{1}{2}}\left(J+\frac{1}{2}\right)\left\langle H i J_{y}\right\rangle_{i-i}\right\}+\frac{8}{3}\left\langle H i J_{y}^{3}\right\rangle_{i-i}- \\
& -2\left(X+2\left\langle J_{y}^{2}\right\rangle_{i i}-\frac{2}{3}\right)\left\langle H i J_{y}\right\rangle_{i-i} \\
& W_{1}^{(3)}=2\left\langle H J_{y}^{4}\right\rangle_{i-i}-4\left\langle J_{y}^{2}\right\rangle_{i-i}\left\langle H J_{y}^{2}\right\rangle_{i i}-\left(4 X+4\left\langle J_{y}^{2}\right\rangle_{i i}-\frac{8}{3}\right)\left\langle H J_{y}^{2}\right\rangle_{i-i} .
\end{aligned}
$$

The functions $W_{0}^{(2)}$ and $W_{1 / 2}^{(2)}$ are already included in equation (28) in a slightly different way. The functions $W^{(4)}$, $W_{i l}$ and $L_{i l}$ have lengthy forms and we do not present them explicity.

Appendix E : Variational equation. - The method of variation of the projected state out of the intrinsic Slater determinant constructed with individual wave functions $u_{i}(\mathbf{r}, \sigma)$ is that of reference [12]. We first define a set of functions $\tilde{u}(r, \sigma ; \beta)$, which are orthonormal to the set of functions $u_{i}$, as

$$
\tilde{u}_{i}(r, \sigma ; \beta)=\sum_{k} \exp \left(-i j_{y} \beta\right) u_{k}(r, \sigma) n_{k i}(\beta),
$$


where $n_{k i}$ is the element of the inverse of the matrix $[N(\beta)]$. With these $u_{i}$, the function $F(u, \beta)$ in equation (41) becomes

with

$$
F(u, \beta)=t \tilde{u}_{i}+\sum_{k}\left\{\left\langle u_{k}\left|V_{12}\right| \tilde{u}_{k} \tilde{u}_{i}\right\rangle-\varepsilon_{i k} \tilde{u}_{k}\right\},
$$

$$
\varepsilon_{i k}=\left\langle u_{k}|t| \tilde{u}_{i}\right\rangle+\sum_{l}\left\langle u_{k} u_{l}\left|V_{12}\right| \tilde{u}_{i} \tilde{u}_{l}\right\rangle+\delta_{i k}\left\{E_{J}-h(\beta)\right\}
$$

Here $E_{J}$ is the projected energy and $h(\beta)=\sum_{i}\left\langle u_{i}|t| \tilde{u}_{i}\right\rangle+\frac{1}{2} \sum_{i k}\left\langle u_{i} u_{k}\left|V_{12}\right| \tilde{u}_{i} \tilde{u}_{k}\right\rangle$. We expand now $F(u, \beta)$ in powers of $\beta$ as

$$
F(u, \beta)=F_{0}(u)+\beta^{2} F_{2}(u)+\beta^{4} F_{4}(u)+\cdots
$$

and $F_{m}(u)$ in inverse powers of $X$ as

$$
F_{m}(u)=F_{m}^{(0)}\left(u^{0}\right)+\frac{1}{X} F_{m}^{(1)}\left(u^{0}, u^{1}\right)+\frac{1}{X^{2}} F_{m}^{(2)}\left(u^{0}, u^{1}, u^{2}\right)+\cdots
$$

Combining these expansions with those of $P_{J}$ and $N(\beta)$, we finally obtain the equation (42).

Appendix F : Operators $\boldsymbol{H}_{m n}$ and $\boldsymbol{J}_{y}^{2}$. - The operators $H_{m n}$ in the Hamiltonian in equation (44) are

$$
\begin{aligned}
& H_{40}=\frac{1}{4} \sum_{i k \alpha \beta} V_{i k, \alpha \beta} a_{i}^{+} a_{k}^{+} b_{\beta}^{+} b_{\alpha}^{+}, \quad H_{04}=\frac{1}{4} \sum_{i k \alpha \beta} V_{\alpha \beta, i k} b_{\alpha} b_{\beta} a_{k} a_{i}, \\
& H_{31}=\frac{1}{2} \sum_{i k l \alpha} V_{i k, l \alpha} a_{i}^{+} a_{k}^{+} b_{\alpha}^{+} a_{l}+\frac{1}{2} \sum_{i \alpha \beta \gamma} V_{i \alpha, \beta \gamma} a_{i}^{+} b_{\gamma}^{+} b_{\beta}^{+} b_{\alpha}, \\
& H_{13}=\frac{1}{2} \sum_{i k l \alpha} V_{i \alpha, k l} a_{i}^{+} b_{\alpha} a_{l} a_{k}+\frac{1}{2} \sum_{i \alpha \beta \gamma} V_{\alpha \beta, i \gamma} b_{\gamma}^{+} b_{\alpha} b_{\beta} a_{i}, \\
& H_{22}=\frac{1}{4} \sum_{i k l m} V_{i k, l m} a_{i}^{+} a_{k}^{+} a_{m} a_{l}+\frac{1}{4} \sum_{\alpha \beta \gamma \delta} V_{\alpha \beta, \gamma \delta} b_{\delta}^{+} b_{\gamma}^{+} b_{\alpha} b_{\beta}+\sum_{i k \alpha \beta} V_{i \alpha, \beta k} a_{i}^{+} b_{\beta}^{+} b_{\alpha} a_{k} .
\end{aligned}
$$

Three operators in equation (46) are

$$
\begin{aligned}
&\left(J_{y}^{2}\right)_{0}=\sum_{i \alpha}\left\langle J_{y}\right\rangle_{i \alpha}\left\langle J_{y}\right\rangle_{\alpha i}, \\
&\left(J_{y}^{2}\right)_{1}=\sum_{i k}\left\{\sum_{l}\left\langle J_{y}\right\rangle_{i l}\left\langle J_{y}\right\rangle_{l k}-\sum_{\alpha}\left\langle J_{y}\right\rangle_{i \alpha}\left\langle J_{y}\right\rangle_{\alpha k}\right\} a_{i}^{+} a_{k}+ \\
&+\sum_{i \alpha}\left\{\sum_{l}\left\langle J_{y}\right\rangle_{i l}\left\langle J_{y}\right\rangle_{l \alpha}-\sum_{\beta}\left\langle J_{y}\right\rangle_{i \beta}\left\langle J_{y}\right\rangle_{\beta \alpha}\right\} a_{i}^{+} b_{\alpha}^{+} \\
&+\sum_{i \alpha}\left\{\sum_{l}\left\langle J_{y}\right\rangle_{\alpha l}\left\langle J_{y}\right\rangle_{l i}-\sum_{\beta}\left\langle J_{y}\right\rangle_{\alpha \beta}\left\langle J_{y}\right\rangle_{\beta i}\right\} b_{\alpha} a_{i} \\
&-\sum_{\alpha \beta}\left\{\sum_{l}\left\langle J_{y}\right\rangle_{\alpha l}\left\langle J_{y}\right\rangle_{l \beta}-\sum_{\gamma}\left\langle J_{y}\right\rangle_{\alpha \gamma}\left\langle J_{y}\right\rangle_{\gamma \beta}\right\} b_{\beta}^{+} b_{\alpha}, \\
&\left(J_{y}^{2}\right)_{2}=\sum_{i k \alpha \beta}\left\langle J_{y}\right\rangle_{i \beta}\left\langle J_{y}\right\rangle_{k \alpha} a_{i}^{+} a_{k}^{+} b_{\alpha}^{+} b_{\beta}^{+}+\sum_{i k \alpha \beta}\left\langle J_{y}\right\rangle_{\alpha k}\left\langle J_{y}\right\rangle_{\beta i} b_{\alpha} b_{\beta} a_{i} a_{k} \\
&+2 \sum_{i k l \alpha}\left\langle J_{y}\right\rangle_{i l}\left\langle J_{y}\right\rangle_{k \alpha} a_{i}^{+} a_{k}^{+} b_{\alpha}^{+} a_{l}+2 \sum_{i \alpha \beta \gamma}\left\langle J_{y}\right\rangle_{i \alpha}\left\langle J_{y}\right\rangle_{\beta \gamma} a_{i}^{+} b_{\gamma}^{+} b_{x}^{+} b_{\beta} \\
&+2 \sum_{i k l \alpha}\left\langle J_{y}\right\rangle_{i l}\left\langle J_{y}\right\rangle_{\alpha k} a_{i}^{+} b_{\alpha} a_{k} a_{l}+2 \sum_{i \alpha \beta \gamma}\left\langle J_{y}\right\rangle_{\alpha \beta}\left\langle J_{y}\right\rangle_{\gamma i} b_{\beta}^{+} b_{\gamma} b_{x} a_{i} \\
&+\sum_{i k l m}\left\langle J_{y}\right\rangle_{i k}\left\langle J_{y}\right\rangle_{l m} a_{i}^{+} a_{l}^{+} a_{m} a_{k}-\sum_{\alpha \beta \gamma \delta}\left\langle J_{y}\right\rangle_{\alpha \beta}\left\langle J_{y}\right\rangle_{\gamma \delta} b_{\beta}^{+} b_{\delta}^{+} b_{\alpha} b_{\gamma} \\
&+2 \sum_{i k \alpha \beta}\left\{\left\langle J_{y}\right\rangle_{i \beta}\left\langle J_{y}\right\rangle_{\alpha k}-\left\langle J_{y}\right\rangle_{\alpha \beta}\left\langle J_{y}\right\rangle_{i k}\right\} a_{i}^{+} b_{\beta}^{+} b_{x} a_{k} .
\end{aligned}
$$


Appendix G : $E_{K}^{(1)+}$ for one-particle one-hole state. - When $|n K\rangle=a_{p}^{+} b_{\alpha}^{+}|0\rangle$, we get

$$
\begin{aligned}
& E_{K}^{(1)+}=2 \sum_{i} V_{p \alpha, i \alpha}\left\{\sum_{r}\left\langle J_{y}\right\rangle_{i r}\left\langle J_{y}\right\rangle_{r p}-\sum_{\rho}\left\langle J_{y}\right\rangle_{i \rho}\left\langle J_{y}\right\rangle_{\rho p}\right\}- \\
&-2 \sum_{\beta} V_{p \beta, p \alpha}\left\{\sum_{r}\left\langle J_{y}\right\rangle_{\alpha r}\left\langle J_{y}\right\rangle_{r \beta}-\sum_{\rho}\left\langle J_{y}\right\rangle_{\alpha \rho}\left\langle J_{y}\right\rangle_{\rho \beta}\right\} \\
&+4 \sum_{i \beta} V_{p \beta, i \alpha}\left\{\left\langle J_{y}\right\rangle_{i \beta}\left\langle J_{y}\right\rangle_{\alpha p}-\left\langle J_{y}\right\rangle_{\alpha \beta}\left\langle J_{y}\right\rangle_{i p}\right\} \\
&+2 \sum_{i \beta}\left\{V_{p \beta, i p}+V_{\alpha \beta, \alpha i}+V_{p \alpha, p i} \delta_{\alpha \beta}-V_{\alpha \beta, \alpha p} \delta_{p i}\right\}\left\{\sum_{r}\left\langle J_{y}\right\rangle_{i r}\left\langle J_{y}\right\rangle_{r \beta}-\sum_{\rho}\left\langle J_{y}\right\rangle_{i \rho}\left\langle J_{y}\right\rangle_{\rho \beta}\right\} \\
&-4 \sum_{i k \beta}\left\{V_{p \beta, i k}-V_{p \alpha, i k} \delta_{\alpha \beta}+V_{\alpha \beta, \alpha i} \delta_{k p}\right\}\left\langle J_{y}\right\rangle_{i p}\left\langle J_{y}\right\rangle_{k \beta} \\
&+4 \sum_{i \beta \gamma}\left\{V_{\beta \gamma, i \alpha}-V_{p \beta, p i} \delta_{\gamma \alpha}-V_{\beta \gamma, p \alpha} \delta_{i p}\right\}\left\langle J_{y}\right\rangle_{i \gamma}\left\langle J_{y}\right\rangle_{\alpha \beta} .
\end{aligned}
$$

These interactions are illustrated in figure 6.

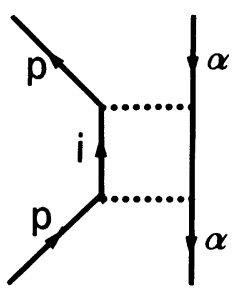

a
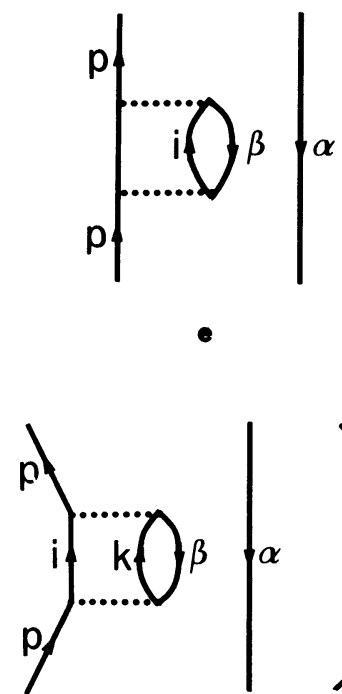

i

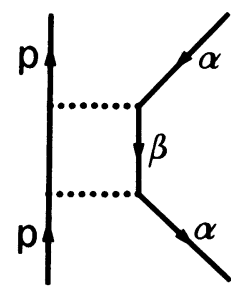

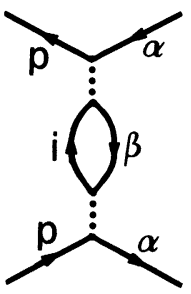

c

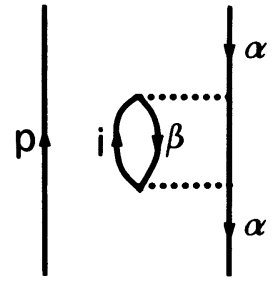

t

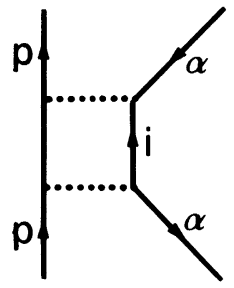

g

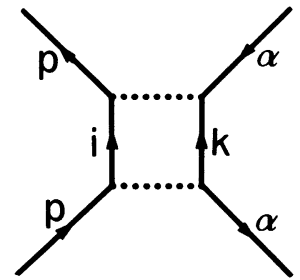

j

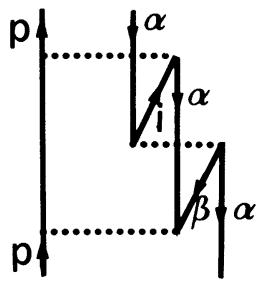

m

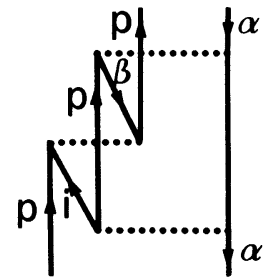

k

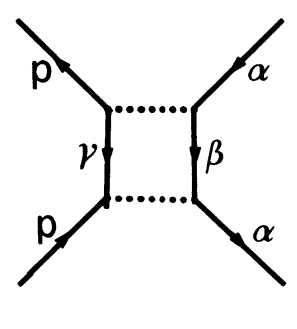

n

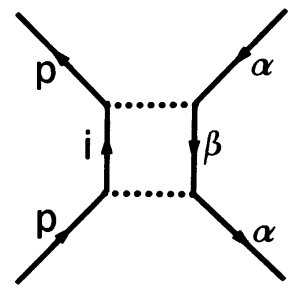

d

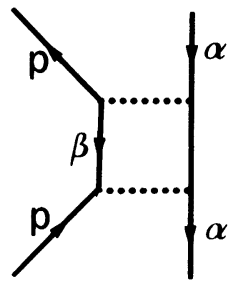

h

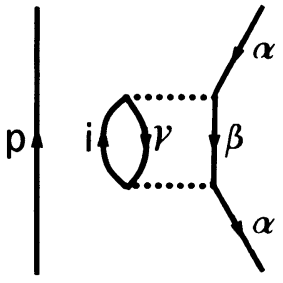

I

Fig. 6. - The diagram representation of $E_{K}^{(1)+}$ for the one-particle one-hole states, $|n K\rangle=a_{p}^{+} b_{\alpha}^{+}|0\rangle$. 
Appendix $H$ : Band-mixing between one-particle one-hole states. - When two bands $K_{i}$ and $K_{l}$ are both represented by one-particle one-hole states such as $\left|n_{i} K_{i}\right\rangle=a_{p}^{+} b_{\alpha}^{+}|0\rangle$ and $\left|n_{l} K_{l}\right\rangle=a_{q}^{+} b_{\beta}^{+}|0\rangle$, the band-mixing term for $|\Delta K|=1$ or $K_{i}+K_{l}=1$ is given by

$$
\begin{aligned}
& \frac{J(J+1)-K_{i} K_{l}}{X^{2}\left(E_{K_{i}}^{(0)}-E_{K_{l}}^{(0)}\right)} \mid \sum_{l \neq p}\left[V_{p \beta, \alpha l}\left\langle i J_{y}\right\rangle_{l q}+V_{p \beta, l q}\left\langle i J_{y}\right\rangle_{l \alpha}+V_{l \beta, \alpha q}\left\langle i J_{y}\right\rangle_{p l}+\right. \\
& \left.+V_{p l, \alpha q}\left\langle i J_{y}\right\rangle_{\beta l}\right]-\sum_{\gamma \neq \alpha}\left[V_{p \gamma, \alpha q}\left\langle i J_{y}\right\rangle_{\beta \gamma}+V_{\gamma \beta, \alpha q}\left\langle i J_{y}\right\rangle_{p \gamma}+V_{p \beta, \gamma q}\left\langle i J_{y}\right\rangle_{\gamma \alpha}+V_{p \beta, \alpha \gamma}\left\langle i J_{y}\right\rangle_{\gamma q}\right] \\
& +\sum_{l, \gamma}\left\{\left[V_{p \gamma, q l} \delta_{\alpha \beta}-V_{\gamma \beta, l \alpha} \delta_{p q}\right]\left\langle i J_{y}\right\rangle_{l \gamma}+\left[-V_{l \beta, \gamma \alpha} \delta_{p q}+V_{p l, q \beta} \delta_{\alpha \beta}\right]\left\langle i J_{y}\right\rangle_{\gamma l}\right\} \\
& +\delta_{K_{i}}\left(\begin{array}{l}
0 \\
1
\end{array}\right) \delta_{K_{l}}\left(\begin{array}{l}
0 \\
1
\end{array}\right)(-1)^{J+1} \text { [the same terms as before but with } \bar{\beta} \text { and } \bar{q} \text { in place of } \beta \text { and } q \text { ] }\left.\right|^{2} \text {. }
\end{aligned}
$$

Appendix I : Matrix element of $\mathrm{HiJ}_{\mathbf{y}}^{3}$. - The matrix element of the operator $\mathrm{HiJ}_{\mathbf{y}}^{3}$ between the one-particle states $\left|n_{i} \pm K_{i}\right\rangle=a_{p(\bar{p})}^{+}|0\rangle$ is

$$
\begin{aligned}
\left\langle H i J_{y}^{3}\right\rangle_{i-i} & =\sum_{k \alpha} V_{p \alpha, \overline{p k}}\left\{3\left\langle i J_{y}\right\rangle_{k \alpha} \sum_{l \beta}\left\langle J_{y}\right\rangle_{l \beta}\left\langle J_{y}\right\rangle_{\beta l}-2 \sum_{l \beta}\left\langle i J_{y}\right\rangle_{k l}\left\langle J_{y}\right\rangle_{l \beta}\left\langle J_{y}\right\rangle_{\beta \alpha}-\right. \\
& \left.-2 \sum_{i \beta}\left\langle i J_{y}\right\rangle_{k \beta}\left\langle J_{y}\right\rangle_{\beta l}\left\langle J_{y}\right\rangle_{l \alpha}+\sum_{l m}\left\langle i J_{y}\right\rangle_{k l}\left\langle J_{y}\right\rangle_{l m}\left\langle J_{y}\right\rangle_{m \alpha}+\sum_{\gamma \beta}\left\langle i J_{y}\right\rangle_{k \beta}\left\langle J_{y}\right\rangle_{\beta \gamma}\left\langle J_{y}\right\rangle_{\gamma \alpha}\right\} \\
& +\sum_{k l \alpha} V_{p \alpha, k l}\left\{3\left\langle i J_{y}\right\rangle_{l \alpha}\left[\sum_{m}\left\langle J_{y}\right\rangle_{k m}\left\langle J_{y}\right\rangle_{m \bar{p}}-\sum_{\gamma}\left\langle J_{y}\right\rangle_{k \gamma}\left\langle J_{y}\right\rangle_{\gamma \bar{p}}\right]\right. \\
& \left.+3\left\langle i J_{y}\right\rangle_{k \bar{p}}\left[\sum_{m}\left\langle J_{y}\right\rangle_{l m}\left\langle J_{y}\right\rangle_{m \alpha}-\sum_{y}\left\langle J_{y}\right\rangle_{l y}\left\langle J_{y}\right\rangle_{\gamma \alpha}\right]\right\} \\
& -\sum_{k \alpha \beta} V_{\beta \alpha, \bar{p} k}\left\{3\left\langle i J_{y}\right\rangle_{k \alpha}\left[\sum_{m}\left\langle J_{y}\right\rangle_{p m}\left\langle J_{y}\right\rangle_{m \beta}-\sum_{\gamma}\left\langle J_{y}\right\rangle_{p \gamma}\left\langle J_{y}\right\rangle_{\gamma \beta}\right]\right. \\
& \left.+3\left\langle i J_{y}\right\rangle_{p \beta}\left[\sum_{m}\left\langle J_{y}\right\rangle_{k m}\left\langle J_{y}\right\rangle_{m \alpha}-\sum_{\gamma}\left\langle J_{y}\right\rangle_{k \gamma}\left\langle J_{y}\right\rangle_{\gamma \alpha}\right]\right\} \\
& +3 \sum_{k l \alpha \beta} V_{\beta \alpha, k l}\left\{\left\langle i J_{y}\right\rangle_{p \alpha}\left\langle J_{y}\right\rangle_{l \beta}\left\langle J_{y}\right\rangle_{k \bar{p}}-\left\langle i J_{y}\right\rangle_{p \beta}\left\langle J_{y}\right\rangle_{l \alpha}\left\langle J_{y}\right\rangle_{k \bar{p}}+\left\langle i J_{y}\right\rangle_{p \bar{p}}\left\langle J_{y}\right\rangle_{k \beta}\left\langle J_{y}\right\rangle_{l \alpha}\right\}
\end{aligned}
$$

\section{References}

[1] Peierls, R. E. and Yoccoz, J., Proc. Phys. Soc. (London) A 70 (1957) 381 ;

Yoccoz, J., Doctoral thesis, Université de Paris (1956) unpublished.

[2] Yoccoz, J., Proc. Phys. Soc. (London) A 70 (1957) 388.

[3] Yoccoz, J., C. R. Hebd. Séan. Acad. Sci. 244 (1957) 1484 ; ibid. 244 (1957) 3052.

[4] Villars, F., Proc. Int. School of Phys. « Enrico Fermi », edited by Bloch C., Vol. XXXVI (Academic Press, New York) 1966, p. 14.

[5] Kelson, I. and Levinson, C. A., Phys. Rev. 134 (1964) B 269 ;

Bassichis, W. H., Levinson, C. A. and Kelson, I., Phys. Rev. 136 (1964) B 380;

Bar-Touv, J. and Kelson, I., Phys. Rev. 138 (1969) B 1035 ;

Bassichis, W. H. and RIPKA, G., Phys. Lett. 15 (1965) 320 ;

Bassichis, W. H., Giraud, B. and RipKa, G., Phys. Rev. Lett. 15 (1965) 930 ;
Bassichis, W. H. and Svenne, J. P., Phys. Rev. Lett. 18 (1967) 80.

[6] Kelson, I., Phys. Lett. 16 (1965) 143.

[7] RIPKA, G., Lectures in Theoretical Physics, Vol. VIII C, edited by Kunz P. D., Lind D. A. and Brittin W. E. (University of Colorado Press, Boulder) 1966, p. 237 ; Fundamentals in Nuclear Theory, edited by De Shalit A. and Villi C. (IAEA, Vienna) 1967, p. 623; Advances in Nuclear Physics, Vol. 1, edited by Baranger M. and Vogt E. (Plenum Press, New York) 1968, p. 183.

[8] Warke, C. S. and Gunye, M. R., Phys. Rev. Lett. 18 (1967) 80 ;

Gunye, M. R. and WARKe, C. S., Phys. Rev. 156 (1967) 1087 ; ibid. 159 (1967) 885.

[9] Johnstone, I. P. and Benson, H. G., Nucl. Phys. A 134 (1969) 68.

[10] Brut, F. and Jang, S., Phys. Rev. C 14 (1976) 1638 ; BRUt, F., Mankoc-Borstnik, N. and JANG, S., Nucl. Phys. A 304 (1978) 429;

Brut, F. and JANG, S., Phys. Lett. 77B (1978) 355 ; 
Mankoc-Borstnik, N., Brut, F. and JANG, S., Nucl. Phys. A 325 (1979) 100 ;

Brut, F., Mankoc-Borstnik, N. and JANG, S., J. Phys. G : Nucl. Phys. 5 (1979) 1249.

[11] Peierls, R. E. and Thouless, D. J., Nucl. Phys. 38 (1962) 154.

[12] Yoccoz, J., Proc. Int. School of Phys. « Enrico Fermi », edited by Bloch C., Vol. XXXVI (Academic Press, New York) 1966, p. 474 ;

Rouhaninejad, H. and Yoccoz, J., Nucl. Phys. 78 (1966) 353.

[13] ZEH, H. D., Z. Phys. 188 (1965) 361 ; ibid. 202 (1967) 38.

[14] Kamlah, A., Z. Phys. 216 (1968) 52.

[15] Beck, R., Mang, H. J. and Ring, P., Z. Phys. 231 (1970) 26 ;

RING, P., BeCK, R. and MaNG, H. J., ibid. 231 (1970) 10 ;

Mang, H. J., Phys. Rep. 18, no 6 (1975) 325 ;

Islam, S., Mang, H. J. and Ring, P., Nucl. Phys. A 326 (1969) 161.

[16] Onishi, N., Prog. Theor. Phys. 40 (1968) 84.

[17] Villars, F. and Schemeing-Rogerson, N., Ann. Phys. 63 (1971) 443.

[18] Gunye, M. R. and Warke, C. S., Phys. Rev. C 20 (1979) 372.

[19] Parikh, J. C. and Rowe, D. J., Phys. Rev. 175 (1968) 1293 ;

Ullah, N. and Rowe, D. J., Phys. Rev. 188 (1969) 1640 ;

Castel, B. and Parikh, J. C., Phys. Rev. C 1 (1970) 990 ;
Rowe, D. J., Ullah, N., Wong, S. S. M., Parikh, J. C. and Castel, B., Phys. Rev. C 3 (1971) 73.

[20] Rowe, D. J., Nuclear Collective Motion (Methuen and Co., London) 1970.

[21] Satpathy, L. and Nair, S. C. K., Phys. Lett. 26B (1968) 257.

[22] Bohr, A. and Mottelson, B. R., Nuclear Structure, Vol. 2 (Benjamin Reading, Massachusetts) 1975; Mat. Fys. Medd. Dan. Vid. Selsk. 27 (1953), $\mathrm{n}^{\mathrm{o}} 16$; ibid. 30 (1955) $\mathrm{n}^{\circ} 1$.

[23] VerhaAr, B. J., Nucl. Phys. 41 (1963) 53 ; ibid. 45 (1963) 129 ; ibid. 54 (1964) 641.

[24] Petry, A. M., Thèse 3e cycle, Université de Grenoble (1970), unpublished.

[25] Rowe, D. J., Nucl. Phys. 61 (1965) 1.

[26] Bhatia, K. L. and De Takacsy, N., Phys. Rev. 187 (1969) 1347.

[27] Bahduri, R. K. and Das GuPta, S., Nucl. Phys. A 212 (1973) 18.

[28] Ullah, N., J. Phys. G. : Nucl. Phys. 5 (1969) 67 ; see also reference [27].

[29] Inglis, D. R., Phys. Rev. 96 (1954) 1059; ibid. 97 (1955) 70 .

[30] CHI-Yu Hu, Nucl. Phys. 66 (1965) 449.

[31] Thouless, D. J., Nucl. Phys. 21 (1960) 225 ;

Thouless, D. J. and Valatin, J. G., Nucl. Phys. 31 (1962) 211.

[32] Kerman, A. K., Mat. Fys. Medd. Dan. Vid. Selsk. 30 (1956) no 15. 